\title{
MULTIRESOLUTION AND WAVELETS
}

\author{
by RONG-QING JIA* $†$ and ZUOWEI SHEN†
}

(Received 20th August 1992)

\begin{abstract}
Multiresolution is investigated on the basis of shift-invariant spaces. Given a finitely generated shift-invariant subspace $S$ of $L_{2}\left(\mathbb{P}^{d}\right)$, let $S_{k}$ be the $2^{k}$-dilate of $S(k \in \mathbb{Z})$. A necessary and sufficient condition is given for the sequence $\left\{S_{k}\right\}_{k e Z}$ to form a multiresolution of $L_{2}\left(\mathbb{P}^{d}\right)$. A general construction of orthogonal wavelets is given, but such wavelets might not have certain desirable properties. With the aid of the general theory of vector fields on spheres, it is demonstrated that the intrinsic properties of the scaling function must be used in constructing orthogonal wavelets with a certain decay rate. When the scaling function is skew-symmetric about some point, orthogonal wavelets and prewavelets are constructed in such a way that they possess certain attractive properties. Several examples are provided to illustrate the general theory.
\end{abstract}

1980 Mathematics subject classification: 41A63, 41A30, 42C99, 46C99.

\section{Introduction}

In this paper we investigate multiresolution and wavelet decomposition in the multivariate situation. Our study is based on the theory of finitely generated shiftinvariant spaces. Certain basic questions concerning multiresolution are addressed and solved. Orthogonal wavelets are constructed explicitly under almost the weakest assumptions. When the scaling function is skew-symmetric about some point, orthogonal wavelets and prewavelets are constructed in such a way that they possess certain desirable properties.

Refinable shift-invariant spaces constitute an appropriate setting for wavelet theory. Given a linear space $S$ of (complex-valued) functions defined on $\mathbb{R}^{d}$, we say that $S$ is shift-invariant if, for any $s \in S$ and $j \in \mathbb{Z}^{d}$, the shift $s(\cdot-j)$ of $s$ is also in $S$; we say that $S$ is refinable if, for any $s \in S$, its dyadic dilate $s(\cdot / 2)$ is also in $S$. For example, for each $p \in[1, \infty]$, the space $L^{p}\left(\mathbb{R}^{d}\right)$ is refinable and shift-invariant, where $L_{p}\left(\mathbb{R}^{d}\right)$ denotes the Banach space of all measurable functions $f$ such that

$$
\|f\|_{p^{\prime}}:=\left(\int_{R^{d}}|f(x)|^{p} d x\right)^{1 / p}<\infty
$$

For $\phi \in L_{2}\left(\mathbb{R}^{d}\right)$, we define $S(\phi)$ to be the smallest closed shift-invariant subspace of

* Supported in part by NSERC Canada under Grant OGP 121336.

†Supported in part by NATO Collaborative Research under Grant CRG 901018.

¥Supported as a research associate on NSF grant DMS-9000053. 
$L_{2}\left(\mathbb{R}^{d}\right)$ containing $\phi$. We say that $\phi$ is refinable if $S(\phi)$ is. A refinable function is also called a scaling function.

Most methods used in wavelet decompositions rely on the notion of multiresolution as introduced by Mallat [32] and Meyer (see [33]). Here we shall adopt the definition of multiresolution put forward by de Boor, DeVoore, and Ron in [6], which is a generalization of the definition given by Mallat and Meyer. Let $S$ be a shift-invariant subspaces of $L_{2}\left(\mathbb{R}^{d}\right)$. For $k \in \mathbb{Z}$, let $S_{k}$ be the $2^{k}$-dilate of $S$ :

$$
S_{k}=\left\{s\left(2^{k} \cdot\right): s \in S\right\}
$$

We say that $\left\{S_{k}\right\}_{k \in \mathbf{Z}}$ forms a multiresolution of $L_{2}\left(\mathbb{R}^{d}\right)$ if the following conditions are satisfied:

(i) $S_{k} \subset S_{k+1}, k \in \mathbb{Z}$;

(ii) $\overline{\bigcup_{k \in Z} S_{k}}=L_{2}\left(\mathbb{R}^{d}\right)$;

(iii) $\bigcap_{k \in Z} S_{k}=\{0\}$.

Evidently, the condition (i) is equivalent to saying that $S$ is refinable.

In [32] and [33], the space $S$ was assumed to be $S(\phi)$ for some function $\phi \in L_{2}\left(\mathbb{R}^{d}\right)$. Moreover, $\phi$ was assumed to have stable shifts. The concept of stability involves sequences on the lattice $\mathbb{Z}^{\mathrm{d}}$. For each $p \in[1, \infty]$, let $l_{p}\left(\mathbb{Z}^{d}\right)$ be the Banach space of all sequences $a$ on $\mathbb{Z}$ such that

$$
\|a\|_{p}:=\left(\sum_{j \in Z^{d}}|a(j)|^{p}\right)^{1 / p}<\infty
$$

We also denote by $l_{0}\left(\mathbb{Z}^{d}\right)$ the linear space of all finitely supported sequences on $\mathbb{Z}^{d}$. For a function $\phi$ defined on $\mathbb{R}^{d}$ and a sequence $a$ on $\mathbb{Z}^{d}$, we use the semi-convolution notation $\phi^{* \prime} a$ to denote the sum

$$
\sum_{j \in Z^{d}} \phi(\cdot-j) a(j)
$$

whenever this sum makes sense. In particular, $\phi^{* \prime} a$ is well defined if $a \in l_{0}\left(\mathbb{Z}^{d}\right)$. We say that the shifts of a function $\phi \in L_{2}\left(\mathbb{R}^{d}\right)$ are stable if there exist two positive constants $C_{1}$ and $C_{2}$ such that

$$
C_{1}\|a\|_{2} \leqq\left\|\phi^{* \prime} a\right\|_{2} \leqq C_{2}\|a\|_{2} \text { for all } a \in l_{0}\left(\mathbb{Z}^{d}\right) .
$$

The stability condition can be characterized in terms of the Fourier transform of $\phi$. The Fourier transform of $f \in L_{1}\left(\mathbb{R}^{d}\right)$ is defined by

$$
f(\xi):=\int_{\mathbb{R}^{d}} f(x) e^{-i \xi \cdot x} d x, \quad \xi \in \mathbb{R}^{d}
$$


where $i$ denotes the imaginary unit, and $\xi \cdot x$ denotes the inner product of the two vectors $\xi$ and $x$ in $\mathbb{R}^{d}$. The Fourier transform has a natural extension to $L_{2}\left(\mathbb{R}^{d}\right)$. It is well-known (see e.g., [33, Chap. 2]) that (1.1) is equivalent to the following conditions:

$$
C_{1}^{2} \leqq \sum_{\beta \in Z^{d}}|\hat{\phi}(\xi+2 \pi \beta)|^{2} \leqq C_{2}^{2} \quad \text { for almost all } \xi \in \mathbb{R}^{d} .
$$

See the work of Jia and Micchelli ([24] and [25]) for a characterization of the stability of the shifts of a finite number of functions in $L_{p}\left(\mathbb{R}^{d}\right) 1 \leqq p \leqq \infty$. When $p=2$, their results were generalized by de Boor, DeVore, and Ron in [5].

If $\phi \in L_{2}\left(\mathbb{R}^{d}\right)$ has stable shifts, then $\phi * a$ is well defined for any $a \in l_{2}\left(\mathbb{Z}^{d}\right)$, and $S(\phi)$ can be characterized as the space $\left\{\phi * a: a \in l_{2}\left(\mathbb{Z}^{d}\right)\right\}$. In particular, $\phi$ is refinable if and only if $\phi$ satisfies a refinement equation

$$
\phi=\sum_{j \in Z^{d}} b(j) \phi(2 \cdot-j)
$$

where $b \in l_{2}\left(\mathbb{Z}^{d}\right)$ is called the refinement mask. In the case when $d=1$ and the (refinement) mask is finitely supported, the stability of the shifts of $\phi$ was characterized by Jia and Wang [27] in terms of the mask.

Of fundamental importance in multiresolution analyses is the following question. Under what conditions on $\phi$ does $S(\phi)$ generate a multiresolution of $L_{2}\left(\mathbb{R}^{d}\right)$ ? It was proved in [33, Chap. 2, Theorem 5] that $S(\phi)$ generates a multiresolution of $L_{2}\left(\mathbb{R}^{d}\right)$ provided that $\phi$ is refinable, $\phi$ has stable shifts, and $\phi$ satisfies the regularity conditions:

$$
|\phi(x)| \leqq C_{m}(1+|x|)^{m} \quad \text { for all } m \in \mathbb{N} \text { and } x \in \mathbb{R}^{d},
$$

where $\mathbb{N}$ is the set of natural numbers, $|x|$ denotes the Euclidean norm of $x$, and $C_{m}$ are positive constants which might depend on $m$. In the work of Jia and Micchelli [25], the aforementioned regularity conditions were relaxed so that $\phi$ is only required to satisfy

$$
\sum_{j \in \mathbf{Z}^{d}}|\phi(\cdot-j)| \in L_{2}\left([0,1)^{d}\right) .
$$

Various improvements of the above results were made in [31] and [39]. But the ultimate solution to the question concerning multiresolution was obtained by de Boor, DeVore, and Ron in [6]. Their results can be stated as follows.

Theorem 1.1. Let $S=\mathscr{S}(\phi)$ be the shift-invariant subspace generated by a function $\phi \in L_{2}\left(\mathbb{R}^{d}\right)$, and let $S_{k}$ be the $2^{k}$-dilate of $S$ for $k \in \mathbb{Z}$. If, in addition, $\phi$ is refinable, then $\bigcup_{k \in Z} S_{k}=L_{2}\left(\mathbb{R}^{d}\right)$ if and only if

$$
\bigcap_{k \in \mathbf{Z}}\left(2^{k} Z(\hat{\phi})\right)
$$


is a set of measure zero, where $Z(\hat{\phi}):=\left\{\xi \in \mathbb{R}^{d}: \hat{\phi}(\xi)=0\right\}$.

Theorem 1.2. Let $S=\mathscr{S}(\phi)$ for some $\phi \in L_{2}\left(\mathbb{R}^{d}\right)$. Then $\bigcap_{k \in \mathrm{Z}} S_{k}=\{0\}$.

Note that in Theorems 1.1 and $1.2 \phi$ is not assumed to have stable shifts. In fact, it was proved earlier in [25] that the union of $S_{k}(k \in \mathbb{Z})$ is dense in $L_{2}\left(\mathbb{R}^{d}\right)$ provided that $\phi$ is refinable, $\phi$ satisfies $(1.3)$, and $\hat{\phi}(0) \neq 0$.

In this paper we investigate multiresolution induced by finitely generated shiftinvariant (FSI) spaces. A shift-invariant space is said to be finitely generated if it is generated by a finite number of functions. Let $\Phi$ be a finite set of functions in $L_{2}\left(\mathbb{R}^{d}\right)$. By $\mathscr{S}(\Phi)$ we denote the smallest closed shift-invariant subspace of $L_{2}\left(\mathbb{R}^{d}\right)$ containing $\Phi$. We say that $\Phi$ provides an orthonormal (resp. stable) basis for a shift-invariant space $S$ if $\mathscr{S}(\Phi)=S$ and the shifts of the functions in $\Phi$ are orthonormal (resp. stable). As we shall see, Theorem 1.1 has an easy extension to FSI spaces. In the next section we extend Theorem 1.2 to FSI spaces. Note that the method used in [6] to prove Theorem 1.2 is not readily applicable to FSI spaces. Thus we shall take a different approach to this problem.

Given a refinable shift-invariant subspace $S$ of $L_{2}\left(\mathbb{R}^{d}\right)$, we define the wavelet space $W$ as the orthogonal complement of $S_{0}$ in $S_{1}$. It follows that $W_{k}$, the $2^{k}$-dilate of $W$, is the orthogonal complement of $S_{k}$ in $S_{k+1}(k \in \mathbb{Z})$. Thus, in order to find an orthonormal basis for $L_{2}\left(\mathbb{R}^{d}\right)$, it suffices to find an orthonormal basis for the wavelet space $W$. A subset $\Psi$ of $W$ is called an orthogonal wavelet set if $\Psi$ provides an orthonormal basis for $W ; \Psi$ is called a prewavelet set if $\Psi$ provides a stable basis for $W$.

The construction of univariate orthogonal wavelets is relatively simple (see, e.g., [32] and [33]). Let $\phi$ be a function in $L_{2}(\mathbb{R})$ having orthonormal shifts. If $\phi$ satisfies the refinement equation

$$
\phi=\sum_{j \in \mathbf{Z}} b(j) \phi(2 \cdot-j)
$$

then the function

$$
\psi=\sum_{j \in \mathbf{Z}}(-1)^{j} \overline{b(1-j)} \phi(2 \cdot-j)
$$

provides an orthonormal basis for the wavelet space $W$, where we have used the notation $\bar{a}$ to denote the complex conjugate of a complex number $a$. Daubechies in [15] constructed smooth scaling functions of compact support having orthonormal shifts and then applied the above method to obtain smooth orthogonal wavelets of compact support. A construction similar to the one given above was used by Chui and Wang ([7] and [8]), and Micchelli [34] to produce prewavelets.

In the multivariate case $(d>1)$, if the scaling function is a tensor product of univariate functions, then the construction of orthogonal wavelets is also straightforward (see e.g., [40]). However, when the scaling function is not a tensor product of univariate functions, the construction of wavelets is much more complicated than the construction 
of wavelets in one variable. Under the conditions that $\phi$ has stable shifts and satisfies the regularity condition (1.2), Gröchenig [19] first proved the existence of orthogonal wavelet sets (see also [33, p. 92]). Under a weaker condition Jia and Micchelli [26] gave another proof of the existence of orthogonal wavelet sets. But these proofs are not constructive. In [6], assuming $\hat{\phi} \neq 0$ a.e., de Boor, DeVore, and Ron gave an explicit construction of orthogonal wavelet sets. But their construction does not cover all the cases considered in [26]. In Section 3, we will give a simple construction of orthogonal wavelets provided

$$
\sum_{\beta \in Z^{d}}|\hat{\phi}(\xi+2 \beta \pi)|^{2}>0 \quad \text { for almost all } \xi \in \mathbb{R}^{d}
$$

We will also discuss how to construct wavelets when $S$ is generated by a finite number of functions. This discussion extends the previous results in [18] and [35]. Also see [13] and [23] for nonorthogonal wavelet decompositions associated with refinable FSI spaces.

Following the approach of Jia and Micchelli in [25] and [26], we shall establish our results of wavelet decomposition on the basis of the notion of extensibility. See [36] for an exposition of the work of Jia and Micchelli. Section 4 is devoted to a discussion of extensibility. More precisely, we shall reduce the problem of wavelet decomposition into a problem of matrix extension. The latter problem can be formulated as follows. Given a vector whose components are functions on the unit sphere, find a matrix whose entries are also functions on the sphere such that the matrix is always nonsingular, and has the given vector as its first row. If the entries of the matrix are only required to be measurable, then the extension problem can be easily solved. The disadvantage of using merely measurable functions in wavelet decomposition is that usually we can say nothing about the decay of the resulting wavelets. To have some control of the decay of the wavelets, we must require that the entries of the matrix be continuous. Thus, one is led to the deep theory of vector fields on spheres. In view of this theory, we shall see that there is no universal way of constructing wavelets except for some special cases. One exceptional but important class of scaling functions is the class of symmetric functions in the low dimensions $(d \leqq 3)$. For this class of scaling functions Reimenschneider and Shen in [37] and [38] gave a very useful construction of orthogonal wavelet and prewavelet sets. (Also see [9].) Simple constructions of wavelets were given in [30], [39], and [6] for those scaling functions whose Fourier transforms are positive almost everywhere.

The discussion in Section 4 suggests that the intrinsic properties of the scaling functions must be employed in order to construct wavelets with certain decay rates. The property of a scaling function being symmetric not only has physical significance (see [15] and [8]), but also is useful in the construction of wavelets and prewavelets. In Section 5 we shall give an explicit construction of wavelets with certain decay rates and compactly supported prewavelets for scaling functions that are skew-symmetric about some point in $\mathbb{R}^{d}$, where the space dimension $d$ can be arbitrary. In particular, orthogonal wavelets generated by box splines in $\mathbb{R}^{d}$ will be discussed. 


\section{Multiresolution}

In this section we investigate multiresolution of $L_{2}\left(\mathbb{R}^{d}\right)$ based on FSI spaces. We give a complete solution of questions concerning the density of the union and the triviality of the intersection of the dyadic sequence of an FSI space used in multiresolution. This is an extension of the corresponding result in [6] to FSI spaces.

Theorem 1.1 has an easy extension.

Theorem 2.1. Let $S=\mathscr{S}(\Phi)$, where $\Phi$ is a finite subset of $L_{2}\left(\mathbb{R}^{d}\right)$, and let $S_{k}$ be the $2^{k}$-dilate of $S(k \in \mathbb{Z})$. If $\left\{S_{k}\right\}_{k \in Z}$ is a nested sequence, then $\bigcup_{k \in Z} S_{k}=L_{2}\left(\mathbb{R}^{d}\right)$ if and only if

$$
\bigcap_{\phi \in \Phi} \bigcap_{k \in Z}\left(2^{k} Z(\hat{\phi})\right)
$$

is a set of measure zero.

This theorem can be proved in the same way as Theorem 4.2 of [6] was done. One first proves that the space $\bigcup_{k \in Z} S_{k}$ is translation-invariant and then invoke the well-known result of Wiener [41, p. 100] about the characterization of the density of a translation-invariant subspace in $L_{2}\left(\mathbb{R}^{d}\right)$ in terms of its Fourier transform.

Theorem 1.2 can also be extended to FSI spaces.

Theorem 2.2. Let $S=\mathscr{S}(\Phi)$ be an FSI subspace of $L_{2}\left(\mathbb{R}^{d}\right)$. Then

$$
\bigcap_{k \in \mathbf{Z}} S_{k}=\{0\}
$$

Our proof of Theorem 2.2 is based on the following lemma.

Lemma 2.3. Given $\Phi=\left\{\phi_{1}, \ldots, \phi_{n}\right\} \subset L_{2}\left(\mathbb{R}^{d}\right)$, one can find $\Psi=\left\{\psi_{1}, \ldots, \psi_{n}\right\} \subset L_{2}\left(\mathbb{R}^{d}\right)$ such that $S(\Phi) \subseteq S(\Psi)$ and the shifts of $\psi_{1}, \ldots, \psi_{b n}$ are orthonormal.

Proof of Theorem 2.2. Assuming that Lemma 2.3 is valid, we prove Theorem 2.2 as follows (cf. [12], [33], and [39]). By Lemma 2.3, it suffices to prove the theorem for the case when $\Phi=\left\{\phi_{1}, \ldots, \phi_{n}\right\}$ and the shifts of $\phi_{1}, \ldots, \phi_{n}$ are orthonormal.

Let $\boldsymbol{P}_{k}$ be the orthogonal projector from $L_{2}\left(\mathbb{R}^{d}\right)$ onto $S_{k}(k \in \mathbb{Z})$. The theorem will be established if we can prove

$$
P_{k} f \rightarrow 0 \text { as } k \rightarrow-\infty
$$

for every $f \in L_{2}\left(\mathbb{R}^{d}\right)$. Since $\left\|P_{k}\right\|=1$ for all $k \in \mathbb{Z}$, and since compactly supported functions are dense in $L_{2}\left(\mathbb{R}^{d}\right)$, it is sufficient to show that $(2.1)$ is true for any compactly supported function $f \in L_{2}\left(\mathbb{R}^{d}\right)$. Let $f$ be such a function. Then $P_{k} f$ can be expressed as

$$
P_{k} f=\sum_{j=1}^{n} \sum_{\alpha \in \mathbf{Z}^{d}} c_{k, j}(\alpha) 2^{k d / 2} \phi_{j}\left(2^{k} \cdot-\alpha\right),
$$


where $c_{k, j}$ are sequences in $l_{2}\left(\mathbb{Z}^{d}\right)(j=1, \ldots, n)$. Since the shifts of $\phi_{1}, \ldots, \phi_{n}$ are orthonormal, we deduce from the above equality that

$$
\begin{aligned}
\left\|P_{k} f\right\|_{2}^{2} & =\sum_{j=1}^{n}\left\|c_{k, j}\right\|_{2}^{2} \\
& \leqq \sum_{j=1}^{n} \sum_{\alpha \in \mathbf{Z}^{d}}\left(\int_{\mathbf{R}^{d}}\left|f(x) 2^{k d / 2} \phi_{j}\left(2^{k} x-\alpha\right)\right| d x\right)^{2} .
\end{aligned}
$$

If $f$ is supported in the cube $[-R, R]^{d}$ for some $R>0$, then by the Cauchy-Schwartz inequality we have that for $k<0,|k|$ sufficiently large,

$$
\left\|P_{k} f\right\|_{2}^{2} \leqq\|f\|_{2}^{2} \sum_{j=1}^{n} \int_{E_{k}}\left|\phi_{j}(x)\right|^{2} d x
$$

where

$$
E_{k}=\bigcup_{\alpha \in Z^{d}}\left(\alpha+2^{k}[-R, R]^{d}\right)
$$

Now (2.1) follows by letting $k \rightarrow-\infty$ in (2.2).

In order to prove Lemma 2.3 , we first establish an auxiliary result concerning linear homogeneous equations with the coefficients being measurable functions. To this end, let $X$ be a nonempty set, and let $\mathscr{M}$ be a $\sigma$-algebra on $X$. Then $(X, \mathscr{M})$ is a measurable space and the sets in $\mathscr{M}$ are called measurable sets. A function $f: X \rightarrow \mathbb{C}$ is called measurable if the preimage of any open set in $\mathbb{C}$ under $f$ is measurable.

Lemma 2.4. Let $A=\left(a_{j k}\right)_{1 \leqq j \leqq m, 1 \leqq k \leqq n}$ be a matrix of measurable functions on $(X, \mathscr{M})$. If $n>m$, then there exist measurable functions $u_{1}, \ldots, u_{n}$ such that for almost all $x \in X$,

$$
\sum_{k=1}^{n}\left|u_{k}(x)\right|^{2}=1
$$

and

$$
\sum_{k=1}^{n} a_{j k}(x) u_{k}(x)=0, \quad j=1, \ldots, m
$$

Proof. For a subset $J$ of $\{1, \ldots, m\}$ and a subset $K$ of $\{1, \ldots, n\}$,we denote by $A(J, K)$ the matrix $\left(a_{j k}\right)_{j \in J, k \in K}$. To each pair $(J, K)$ we associate a set $E(J, K)$ as follows. If $\# J \neq \# K$, where $\# J$ denotes the number of elements in $J$, let $E(J, K)$ be the empty set; otherwise, let

$$
E(J, K):=\{x \in X: \operatorname{rank}(A(J, K)(x))=\# J=\operatorname{rank}(A(x))\}
$$


It is easily seen that $E(J, K)$ is a measurable set and

$$
X=\bigcup_{(J, K)} E(J, K)
$$

where $J$ and $K$ run over all possible subsets of $\{1, \ldots, m\}$ and $\{1, \ldots, n\}$, respectively. We can find measurable subsets $F(J, K)$ of $E(J, K)$ such that $X$ is the disjoint union of $F(J, K)$. We shall define $u_{1}, \ldots, u_{n}$ piecewise on each set $F(J, K)$. For this purpose we fix a pair $(J, K)$ such that $F(J, K)$ is not empty. Then $\# J=\# K \leqq m$. If \#J=0, then $A(x)$ is the zero matrix for every $x \in F(J, K)$. In this case, set

$$
v_{1}(x):=1 \quad \text { and } \quad v_{2}(x)=\cdots=v_{n}(x):=0 \quad \text { for } x \in F(J, K)
$$

If $\# J \geqq 1$, then we choose an element $l \in\{1, \ldots, n\} \backslash K$. For $x \in F(J, K)$, let $v_{l}(x):=1$, $v_{k}(x):=0$ for $k \in\{1, \ldots, n\} \backslash K \backslash\{l\}$. By Cramer's rule one can determine $v_{k}(x), k \in K$ in a unique way such that for all $j \in J$,

$$
\sum_{k=1}^{n} a_{j k}(x) v_{k}(x)=0
$$

But $\operatorname{rank}(A(x))=\# J$, hence the above equality is true for all $j=1, \ldots, m$. The functions $v_{k}(k=1, \ldots, n)$ so defined are measurable on $F(J, K)$. Now we define $u_{k}(k=1, \ldots, n)$ on $\boldsymbol{X}$ as follows:

$$
u_{k}(x)=v_{k}(x) /\left(\sum_{r=1}^{n}\left|v_{r}(x)\right|^{2}\right)^{1 / 2}, \quad x \in F(J, K)
$$

Since $X$ is the disjoint union of the measurable sets $F(J, K)$, each $u_{k}$ is well defined and is measurable. Clearly, $\left(u_{k}\right)_{1 \leqq k \leqq n}$ satisfies (2.3) and (2.4).

The proof of Lemma 2.3 also relies on the basic theory of shift-invariant spaces. Let us first recall the bracket product notation, which was introduced in [25] and refined in [4]. For $f, g \in L_{2}\left(\mathbb{R}^{d}\right)$, set

$$
[f, g]:=\sum_{\beta \in Z^{d}} f(\cdot+2 \pi \beta) \overline{g(\cdot+2 \pi \beta)}
$$

Then $[f, g]$ is a $2 \pi$-periodic function. Note that $[f, g] \in L_{1}\left([0,2 \pi)^{d}\right)$ as long as $f, g \in L_{2}\left(\mathbb{R}^{d}\right)$. Thus, $[\hat{f}, \hat{g}]$ has a Fourier series expansion:

$$
[\hat{f}, \hat{g}](\xi) \sim \sum_{\alpha \in \mathbf{Z}^{d}} c_{\alpha}(f, g) e^{i \alpha \cdot \xi}, \quad \xi \in[0,2 \pi)^{d}
$$

The Fourier coefficients $c_{a}(f, g)$ can be easily found: 


$$
\begin{aligned}
c_{\alpha}(f, g) & =\frac{1}{(2 \pi)^{d}} \int_{[0,2 \pi)^{d}}[\hat{f}, \hat{g}](\xi) e^{-i \alpha \cdot \xi} d \xi \\
& =\frac{1}{(2 \pi)^{d}} \int_{\mathbf{R}^{d}} \hat{f}(\xi) \overline{\hat{g}(\xi)} e^{-i \alpha \cdot \xi} d \xi \\
& =\langle f, g(\cdot+\alpha)\rangle
\end{aligned}
$$

where $\langle f, g\rangle$ denotes the inner product of $f, g$ in $L_{2}\left(\mathbb{R}^{d}\right)$. Three useful facts can be easily derived from the above discussion. First, the shifts of $f \in L_{2}\left(\mathbb{R}^{d}\right)$ are orthogonal to the shifts of $g \in L_{2}\left(\mathbb{R}^{d}\right)$ if and only if $[\hat{f}, \hat{g}]=0$ a.e.; second, the shifts of $f \in L_{2}\left(\mathbb{R}^{d}\right)$ are orthonormal if and only if $[\hat{f}, \hat{f}]=1$ a.e.; third, if $f \in L_{2}\left(\mathbb{R}^{d}\right)$ does not have orthonormal shifts but $[\hat{f}, \hat{f}]>0$ a.e., then the function $f_{*}$ given by

$$
\hat{f}_{*}=\hat{f} /[\hat{f}, \hat{f}]^{1 / 2}
$$

generates $\mathscr{S}(f)$ and has orthonormal shifts.

We also need the following characterization of PSI spaces (see [20] and [4]). Let $\mathscr{S}(\phi)$ be the PSI space generated by a single function $\phi \in L_{2}\left(\mathbb{R}^{d}\right)$. Then a function $f \in L_{2}\left(\mathbb{R}^{d}\right)$ belongs to $\mathscr{S}(\phi)$ if and only if

$$
\hat{f}=\tau \hat{\phi}
$$

for some $2 \pi$-periodic function $\tau$. In particular, $\phi$ is refinable if and only if

$$
\hat{\phi}(2 \cdot)=n \hat{\phi}
$$

for some $2 \pi$-periodic function $\eta$.

Proof of Lemma 2.3. The proof proceeds by induction on $n$. If $\Phi$ contains only one function $\phi \in L_{2}\left(\mathbb{R}^{d}\right)$, then we define $\psi$ as follows:

$$
\hat{\psi}(\xi):= \begin{cases}\hat{\phi}(\xi) / \sqrt{[\hat{\phi}, \hat{\phi}](\xi)}, \text { if } \quad[\hat{\phi}, \hat{\phi}](\xi)>0 \\ 1, \quad \text { if }[\hat{\phi}, \hat{\phi}](\xi)=0 \text { and } \xi \in[0,2 \pi)^{d} \\ 0, \quad \text { elsewhere. }\end{cases}
$$

Clearly,

$$
[\hat{\psi}, \hat{\psi}](\xi)=1 \text { for almost all } \xi \in \mathbb{R}^{d}
$$

Hence $\psi \in L_{2}\left(\mathbb{R}^{d}\right)$ and the shifts of $\psi$ are orthonormal. Moreover, 


$$
\hat{\phi}=\sqrt{[\hat{\phi}, \hat{\phi}]} \hat{\psi} .
$$

This shows that $\phi \in \mathscr{S}(\psi)$, and hence $\mathscr{S}(\phi) \subseteq \mathscr{S}(\psi)$.

Now let $n>1$ and $\Phi=\left\{\phi_{1}, \ldots, \phi_{n}\right\} \subset L_{2}\left(\mathbb{R}^{d}\right)$. By induction hypothesis, there exist $\psi_{1}, \ldots, \psi_{n-1} \in L_{2}\left(\mathbb{R}^{d}\right)$ such that

$$
\mathscr{P}\left(\phi_{1}, \ldots, \phi_{n-1}\right) \subseteq \mathscr{S}\left(\psi_{1}, \ldots, \psi_{n-1}\right)
$$

and the shifts of $\psi_{1}, \ldots, \psi_{n-1}$ are orthonormal. We may assume that $\phi_{n}$ is orthogonal to $\mathscr{S}\left(\psi_{1}, \ldots, \psi_{n-1}\right)$, for otherwise we may replace $\phi_{n}$ by $\phi_{n}-P \phi_{n}$, where $P \phi_{n}$ is the orthogonal projection of $\phi_{n}$ on the space $\mathscr{S}\left(\psi_{1}, \ldots, \psi_{n-1}\right)$. It follows that

$$
\left[\hat{\phi}_{n}, \hat{\psi}_{j}\right]=0 \text { for } j=1, \ldots, n-1 .
$$

Consider the set

$$
Y:=\left\{\xi \in[0,2 \pi)^{d}:\left[\hat{\phi}_{n}, \hat{\phi}_{n}\right](\xi)=0\right\} .
$$

Evidently, the set $Y$ is measurable. Let

$$
a_{j k}(\xi):=\hat{\psi}_{j}\left(\xi+2 \pi k e_{1}\right), \quad \xi \in Y ; j=1, \ldots, n-1 ; k=1, \ldots, n,
$$

where $e_{1}$ denotes the $d$-vector $(1,0, \ldots, 0)$. Consider the matrix $A=\left(a_{j k}\right)_{1 \leqq j \leqq n-1,1 \leqq k \leqq n}$. In what follows, for a given matrix $B$, we denote by $\bar{B}$ and $B^{T}$ the complex conjugate and the transpose of $B$, respectively. By Lemma 2.4 , we can find measurable functions $v_{1}, \ldots, v_{n}$ on $Y$ such that for almost all $\xi \in Y$,

$$
\sum_{k=1}^{n}\left|v_{k}(\xi)\right|^{2}=1
$$

and

$$
\bar{A}(\xi)\left(v_{1}(\xi), \ldots, v_{n}(\xi)\right)^{T}=0 .
$$

We define a function $g$ on $\mathbb{R}^{d}$ as follows:

$$
g(\xi):=\left\{\begin{array}{lll}
v_{m}(\xi), & \text { if } & \xi \in Y+2 \pi m e_{1} \text { for some } m \in\{1, \ldots, n\} \\
0, & \text { if } & \xi \in \mathbb{R}^{d} \backslash \bigcup_{m=1}^{n}\left(Y+2 \pi m e_{1}\right)
\end{array}\right.
$$

The functions $g$ was constructed in such a way that $g$ is measurable and possesses the following two properties:

(a) $\left[g, \hat{\psi}_{j}\right]=0$ for $j=1, \ldots, n-1$, and

(b) $\sum_{\beta \in \mathbf{Z}^{\mathrm{a}}}|g(\xi+2 \pi \beta)|^{2}=1$ for $\xi \in Y$. 
Now let $\psi_{n}$ be the function defined by the rule

$$
\hat{\psi}_{n}(\xi)= \begin{cases}\hat{\phi}_{n}(\xi) / \sqrt{\left[\hat{\phi}_{n}, \hat{\phi}_{n}\right](\xi)}, & \text { if }\left[\hat{\phi}_{n}, \hat{\phi}_{n}\right](\xi)>0 \\ g(\xi), & \text { elsewhere }\end{cases}
$$

Then $\psi_{n}$ is measurable and $\left[\hat{\psi}_{n}, \hat{\psi}_{n}\right]=1$. Hence $\psi_{n} \in L_{2}\left(\mathbb{R}^{d}\right)$. Moreover, $\left[\hat{\psi}_{n}, \hat{\psi}_{j}\right]=0$ for $j=1, \ldots, n-1$. Thus, the shifts of $\psi_{1}, \ldots, \psi_{n}$ are orthonormal. Finally, since $\hat{\phi}_{n}=$ $\left[\hat{\phi}_{n}, \hat{\phi}_{n}\right]^{1 / 2} \hat{\psi}_{n}$, we have $\phi_{n} \in \mathscr{S}\left(\psi_{n}\right)$. This together with (2.5) implies

$$
\mathscr{S}\left(\phi_{1}, \ldots, \phi_{n-1}, \phi_{n}\right) \subseteq \mathscr{S}\left(\psi_{1}, \ldots, \psi_{n-1}, \psi_{n}\right)
$$

The proof of Lemma 2.3 is complete, and so is that of Theorem 2.2.

Remark 2.5. DeVore and Lucier in [17] quoted [6, Theorem 4.3] differently from what was stated there. Let $\phi \in L_{2}\left(\mathbb{R}^{d}\right)$ and $S=\mathscr{S}(\phi)$. It was claimed in [17] that $\overline{\bigcup_{k \in Z} S_{k}}=L_{2}\left(\mathbb{R}^{d}\right)$ whenever $\phi$ is refinable and, in addition,

$$
[\hat{\phi}, \hat{\phi}]>0 \quad \text { a.e. }
$$

This confusion must be clarified. We point out that the condition (2.6) and the condition

$$
\bigcap_{k \in Z}\left(2^{k} Z(\hat{\phi})\right) \text { is a set of measure zero }
$$

are incomparable. For example, if $\phi$ is defined on $\mathbb{R}$ by the rule

$$
\hat{\phi}(\xi)= \begin{cases}1, & \text { for } \xi \in[0,2 \pi) \\ 0, & \text { otherwise }\end{cases}
$$

Then $\phi$ is a refinable function satisfying the condition $(2.6)$. However, $\bigcap_{k \in Z}\left(2^{k} Z(\hat{\phi})\right)=$ $(-\infty, 0)$ is not a set of measure zero. On the other hand, if $\phi$ is given by the formula

$$
\hat{\phi}(\xi)= \begin{cases}1, & \text { for } \quad \xi \in[-\pi / 2, \pi / 2) \\ 0, & \text { elsewhere }\end{cases}
$$

Then $\phi$ is also refinable. In this case the condition $(2.7)$ is satisfied, but $[\hat{\phi}, \phi](\xi)=0$ for $\xi \in(\pi / 2,3 \pi / 2)$.

Remark 2.6. Multiresolution based on non-dyadic dilations of PSI spaces was investigated in [26] and [31]. Theorems 2.1 and 2.2 can be extended to such a situation. Let $T$ be a nonsingular matrix whose entries are all integers. Assume that the spectral radius of $T^{-1}$ is less than 1 . Let $S$ be an FSI subspace of $L_{2}\left(\mathbb{R}^{d}\right)$ and let 


$$
S_{k}:=\left\{f\left(T^{k} \cdot\right): f \in S\right\}, \quad k \in \mathbb{Z}
$$

Then $\bigcap_{k \in Z} S_{k}=\{0\}$ still holds. To see this, we may assume that $\Phi$ consists of functions whose shifts are orthonormal. Consequently, an argument similar to that used in the proof of Lemma 2.2 tells us that $\bigcap_{k \in Z} S_{k}=\{0\}$. Moreover, if $\left\{S_{k}\right\}_{k \in Z}$ is a nested sequence, then $\bigcup_{k \in Z} S_{k}$ is translation-invariant. This can be proved in the same way as Theorem 4.2 of [6] was done. Thus, we can invoke a well-known result of Wiener [40, p. 100] to conclude that $\overline{\bigcup_{k \in Z} S_{k}}=L_{2}\left(\mathbb{R}^{d}\right)$ if and only if

$$
\bigcap_{\phi \in \Phi} \bigcap_{k \in Z}\left(T^{k} Z(\hat{\phi})\right) \quad \text { is a set of measure zero. }
$$

\section{Wavelet decompositions}

This section is devoted to a study of the existence and construction of orthogonal wavelets. We shall give an explicit construction of orthonormal wavelet bases for $L_{2}\left(\mathbb{R}^{d}\right)$, provided that the conditions (2.6) and (2.7) are satisfied.

Let $\phi$ be a function in $L_{2}\left(\mathbb{R}^{d}\right)$. As before, $\mathscr{S}(\phi)$ denotes the PSI space generated by $\phi$, and $S_{k}$ is the $2^{k}$-dilate of $\mathscr{S}(\phi)(k \in \mathbb{Z})$. We assume that $\phi$ is refinable, i.e., $S_{0} \subset S_{1}$. The wavelet space $W$ is defined as the orthogonal complement of $S_{0}$ in $S_{1}$. Our goal is to find a subset $\Psi$ of $W$ such that $\Psi$ provides an orthonormal basis for $W$. If $\Psi$ is such a set, and if $\left\{S_{k}\right\}_{k \in Z}$ forms a multiresolution of $L_{2}\left(\mathbb{R}^{d}\right)$, then

$$
\left\{2^{k d / 2} \psi\left(2^{k} \cdot-\alpha\right): \psi \in \Psi, k \in \mathbb{Z}, \alpha \in \mathbb{Z}^{d}\right\}
$$

forms an orthonormal basis for $L_{2}\left(\mathbb{R}^{d}\right)$.

There has been an extensive study of the existence of orthogonal wavelet sets (see Section 1 for a brief discussion of the related literature). In the multivariate case, Jia and Micchelli ([25] and [26]) considered the construction of orthogonal wavelets and prewavelets under the conditions that the function $\phi$ has stable shifts and the periodization

$$
|\phi|^{0}:=\sum_{\alpha \in Z^{d}}|\phi(\cdot-\alpha)|
$$

of $|\phi|$ is in $L_{2}\left([0,1)^{d}\right)$. de Boor, DeVore, and Ron in [6], investigated this problem under the assumption that $\hat{\phi} \neq 0$ almost everywhere. Their assumption is too restrictive for some interesting wavelets such as the Meyer wavelet (see [16, p. 137]) whose Fourier transform is compactly supported. Moreover, their study does not cover the results of [25] and [26]. Here is an example. Let $\phi$ be a function on $\mathbb{R}$ defined by its Fourier transform:

$$
\hat{\phi}(\xi)= \begin{cases}1-3 /(4 \pi)|\xi|, & \text { for }|\xi| \leqq 4 \pi / 3 \\ 0, & \text { elsewhere }\end{cases}
$$


Then $\hat{\phi}$ is compactly supported, but $\phi$ satisfies all the conditions required in [25].

In our opinion, (2.6) is an appropriate condition for the wavelet decomposition. We assume that (2.6) holds throughout this section. Let $\phi_{*}$ be the function given by

$$
\hat{\phi}_{*}=\hat{\phi} /[\hat{\phi}, \hat{\phi}]^{1 / 2} \text {. }
$$

Then $\mathscr{S}\left(\phi_{*}\right)=\mathscr{S}(\phi)$ and $\phi_{*}$ has orthonormal shifts. Thus, without loss of any generality, we may assume from the beginning that $\phi$ has orthonormal shifts.

Let $g$ be a $2 \pi$-periodic function whose restriction to $[0,2 \pi)^{d}$ belongs to $L_{1}$. Then $g$ can be expanded into a Fourier series. We simply write

$$
g(\xi)=\sum_{\alpha \in \mathbf{Z}^{d}} a(\alpha) e^{i \alpha \cdot \xi}, \quad \xi \in \mathbb{R}^{d},
$$

to indicate the fact that the sum on the right-hand side is the Fourier series of $g$. In particular, if $a \in l_{2}\left(\mathbb{Z}^{d}\right)$, then there exists a unique function $g$ such that (3.1) holds, and in this case the sum on the right-hand of (3.1) converges to $g$ in the $L_{2}$-norm. Often we write (3.1) in another form:

$$
p(z)=\sum_{\alpha \in \mathbf{Z}^{d}} a(\alpha) z^{\alpha}, \quad z \in \mathbb{T}^{d},
$$

where $\mathbb{T}^{d}$ is the $d$-torus

$$
\left\{\left(z_{1}, \ldots, z_{\mathrm{d}}\right) \in \mathbb{C}^{\mathrm{d}}:\left|z_{1}\right|=\cdots=\left|z_{\mathrm{d}}\right|=1\right\},
$$

and $p$ is the function on $\mathbb{T}^{d}$ defined by $p\left(e^{i \xi}\right)=g(\xi)$ for $\xi \in \mathbb{R}^{d}$. We say that $p$ is the symbol of $a$, and $a$ the coefficient sequence in the Laurent expansion of $p$. The symbol of a sequence $a$ is often denoted by $\tilde{a}$.

Let $\mathscr{E}=\mathscr{E}_{d}$ be the set of all extreme points of the unit cube $[0,1]^{d}$, i.e.,

$$
\mathscr{E}=\mathscr{E}_{d}:=\left\{\left(v_{1}, \ldots, v_{\mathrm{d}}\right): v_{j}=0 \text { or } 1 \text { for all } j\right\} .
$$

This affects a decomposition of the lattice $\mathbb{Z}^{d}$ into $2^{d}$ sublattices $2 \mathbb{Z}^{d}+\mu(\mu \in \mathscr{E})$. Since $\phi$ is refinable, it satisfies a refinement equation

$$
\phi=\sum_{\alpha \in \mathbf{Z}^{d}} b(\alpha) \phi(2 \cdot-\alpha)
$$

where $b \in l_{2}\left(\mathbb{Z}^{d}\right)$ is the refinement mask. Let

$$
\phi_{\mu}=\phi(2 \cdot-\mu), \quad \mu \in \mathscr{E} .
$$

Then $S_{1}=\mathscr{P}(\Phi)$, where $\Phi=\left\{\phi_{\mu}: \mu \in \mathscr{E}\right\}$. Using the functions $\phi_{\mu}(\mu \in \mathscr{E})$, we can rewrite (3.2) as 


$$
\phi=\sum_{\mu \in \mathcal{E}} \phi_{\mu} * b_{\mu}
$$

where $b_{\mu}(\mu \in \mathscr{E})$ are the sequences in $l_{2}\left(\mathbb{Z}^{d}\right)$ given by

$$
b_{\mu}(\beta)=b(\mu+2 \beta), \quad \beta \in \mathbb{Z}^{d}
$$

Taking the Fourier transforms of both sides of (3.3), we obtain

$$
\hat{\phi}(\xi)=\sum_{\mu \in \varangle} 2^{d / 2} \hat{\phi}_{\mu}(\xi) p_{\mu}\left(e^{-i \xi}\right), \quad \xi \in \mathbb{R}^{d}
$$

where $p_{\mu}(\mu \in \mathscr{E})$ are the Laurent series given by

$$
p_{\mu}(z):=\sum_{\beta \in \mathbf{Z}^{d}} 2^{-d / 2} b_{\mu}(\beta) z^{\beta}, \quad z \in \mathbb{T}^{d} .
$$

Since $\phi$ has orthonormal shifts, $[\hat{\phi}, \hat{\phi}]=1$ a.e., so it follows that

$$
\left[2^{d / 2} \hat{\phi}_{\mu}, 2^{d / 2} \hat{\phi}_{v}\right]=\delta_{\mu v} \quad \text { a.e. }
$$

where $\delta_{\mu \nu}$ denotes the Kronecker symbol. We can deduce from (3.4) and (3.5) that

$$
1=\sum_{\mu \in \&}\left|p_{\mu}\left(e^{-i \xi}\right)\right|^{2} \quad \text { for almost all } \xi \in \mathbb{R}^{d}
$$

Consequently, $p_{u} \in L_{\infty}\left(\mathbb{T}^{d}\right)$ for all $\mu \in \mathscr{E}$. Suppose we are given $2^{d}$ functions $\psi_{\mu} \in S_{1}(\mu \in \mathscr{E})$. Each $\psi_{\mu}$ has a representation of the form

$$
\psi_{\mu}=\sum_{v \in \delta} 2^{d / 2} \phi_{v} * b_{\mu v}
$$

where $b_{\mu v} \in l_{2}\left(\mathbb{Z}^{d}\right)(\mu, v \in \mathscr{E})$. Let

$$
p_{\mu v}(z):=\sum_{\beta \in Z^{d}} b_{\mu \nu}(\beta) z^{\beta}, \quad z \in \mathbb{J}^{d}
$$

The following theorem plays a prominent role in wavelet decompositions (see [33, p. 84] and [25, Theorem 7.1]).

Theorem 3.1. The set $\left\{\psi_{\mu}(\cdot-\alpha): \mu \in \mathscr{E}, \alpha \in \mathbb{Z}^{d}\right\}$ forms an orthonormal basis for $S_{1}$ if and only if $\left(p_{\mu v}(z)\right)_{\mu, v \in G}$ is a unitary matrix for almost every $z \in \mathbb{J}^{d}$.

Theorem 3.1 tells us a general procedure to find an orthogonal wavelet set. 
Algorithm 3.2. Let $\phi$ be a function in $L_{2}\left(\mathbb{R}^{d}\right)$ having orthonormal shifts. Suppose that $\phi$ satisfies the refinement equation (3.2) with $b$ as its mask.

Step 1. Find $p_{\mu}(\mu \in \mathscr{E})$ by the equation $p_{\mu}(z):=\sum_{\beta \in Z^{d}} 2^{-d / 2} b(2 \beta+\mu) z^{\beta}, z \in \mathbb{J}^{d}$.

Step 2. Find $p_{\mu \nu} \in L_{\infty}\left(\mathbb{T}^{d}\right)(\mu, v \in \mathscr{E})$ such that $p_{0 \nu}=p_{v}$ for all $\nu \in \mathscr{E}$ and that the matrix $\left(p_{\mu v}(z)\right)_{\mu, v \in \varepsilon}$ is unitary for almost every $z \in \mathbb{J}^{d}$.

Step 3. Expand $p_{\mu v}$ into the Laurent series: $p_{\mu v}(z)=\sum_{\alpha \in Z^{d}} b_{\mu v}(\alpha) z^{\alpha}$.

Step 4. Set $\psi_{\mu}:=\sum_{v \in \mathscr{E}} 2^{d / 2} \phi_{v} * b_{\mu v}$ for all $\mu \in \mathscr{E} \backslash\{0\}$, where $\phi_{v}=\phi(2 \cdot-v), v \in \mathscr{E}$. Then $\left\{\psi_{\mu}(\cdot-\alpha): \mu \in \mathscr{E} \backslash\{0\}, \alpha \in \mathbb{Z}^{d}\right\}$ forms an orthonormal basis for the wavelet space $W$.

The above algorithm reduces the problem of wavelet decomposition into the problem of completing a unitary matrix with the first row given. In order to investigate this matrix problem, we first recall some basic facts about Househoulder matrices (see, e.g., $[10$, p. 152]).

Given a matrix $A$, its (complex) conjugate traspose is denoted by $A^{*}$. If $v$ is an $n \times 1$ vector in $\mathbb{C}^{n}$, then $v v^{*}$ is an $n \times n$ matrix, and $v^{*} v$ is a nonnegative number. For a nonzero $n \times 1$ vector $v$ in $\mathbb{C}^{n}$. let $H(v)$ be the Householder matrix given by

$$
H(v):=I-2 v v^{*} / v^{*} v
$$

where $I$ denotes the $n \times n$ identity matrix. It is easily verified that $H(v)$ is both Hermitian and unitary.

Lemma 3.3. Let $a=\left(a_{1}, \ldots, a_{n}\right)^{T}$ be a nonzero vector in $\mathbb{C}^{n}$. Let $\omega \in \mathbb{R}$ be such that $a_{1}=e^{i \omega}\left|a_{1}\right|$. Then

$$
H\left(a+\|a\|_{2} e^{i \omega} e_{1}\right) a=-\|a\|_{2} e^{i \omega} e_{1},
$$

where $e_{1}$ is the $n \times 1$ vector $(1,0, \ldots, 0)^{T}$, and $\|a\|_{2}:=\left(\sum_{j=1}^{n}\left|a_{j}\right|^{2}\right)^{1 / 2}$.

Proof. Write $v:=a+\|a\|_{2} e^{i \omega} e_{1}$. Then

$$
H(v) a=a-2 v\left(v^{*} a\right) /\left(v^{*} v\right) .
$$

We have

$$
\begin{aligned}
v^{*} v & =\left(a^{*}+\|a\|_{2} e^{-i \omega} e_{1}^{*}\right)\left(a+\|a\|_{2} e^{i \omega} e_{1}\right) \\
& =2\|a\|_{2}\left(\|a\|_{2}+\left|a_{1}\right|\right) \\
& =2 v^{*} a .
\end{aligned}
$$

It follows that 


$$
H(v) a=a-v=-\|a\|_{2} e^{i \omega} e_{1}
$$

This lemma is of particular interest when $\|a\|_{2}=1$. In this case,

$$
H(v) a=-e^{i \omega} e_{1}
$$

Since $H(v)$ is a unitary matrix, $H(v)^{-1}=H(v)^{*}$, so

$$
\left(a_{1}, \ldots, a_{n}\right)=a^{T}=\left(-H(v)^{-1} e^{i \omega} e_{1}\right)^{T}=\left(-e^{i \omega}, 0, \ldots, 0\right) \overline{H(v)}
$$

Let $\operatorname{diag}\left\{-e^{i \omega}, 1, \ldots, 1\right\}$ denote the $n \times n$ diagonal matrix with $-e^{i \omega}, 1, \ldots, 1$ as its diagonal entries. Then the matrix

$$
A:=\operatorname{diag}\left\{-e^{i \omega}, 1, \ldots, 1\right\} \overline{H(v)}
$$

is unitary and has $\left(a_{1}, \ldots, a_{n}\right)$ as its first row. We may express $e^{i \omega}$ in another form:

$$
e^{i \omega}=\sigma\left(a_{1}\right)
$$

where $\sigma$ is the function on $\mathbb{C}$ given by the rule

$$
\sigma(w)=\left\{\begin{array}{lll}
w /|w|, & \text { if } & w \in \mathbb{C} \backslash\{0\} \\
1, & \text { if } & w=0
\end{array}\right.
$$

Note that $\sigma$ is not a continuous function. Let us compute the $(j, k)$-entry $a_{j k}$ of the matrix $A$. It follows from (3.9) and (3.11) that

$$
a_{j k}=\delta_{j k}-2 \bar{v}_{j} v_{k} / v^{*} v, \quad j>1 .
$$

But $v^{*} v=2\left(1+\left|a_{1}\right|\right)$ in view of (3.10). Thus, we find that for $j>1$

$$
a_{j k}= \begin{cases}-\bar{a}_{j} \sigma\left(a_{1}\right), & \text { for } k=1 \\ \delta_{j k}-\bar{a}_{j} a_{k} /\left(1+\left|a_{1}\right|\right), & \text { for } k>1\end{cases}
$$

The forgoing discussion is summarized in the following theorem.

Theorem 3.4. Given $\left(a_{1}, \ldots, a_{n}\right) \in \mathbb{C}^{n}$ with $\sum_{k=1}^{n}\left|a_{k}\right|^{2}=1$. Let $a_{1 k}=a_{k}(k=1, \ldots, n)$ and let $a_{j k}$ be given as in (3.12) when $j>1$. Then $\left(a_{j k}\right)_{j, k=1 \ldots, n}$ is a unitary matrix.

Theorem 3.4 yields an explicit method of wavelet decomposition described in the following.

Theorem 3.5. Let $\phi$ be a function in $L_{2}\left(\mathbb{R}^{d}\right)$ satisfying the refinement equation (3.2) and 
having orthonormal shifts. Then the functions $\psi_{\mu}(\mu \in \mathscr{E} \backslash\{0\})$ produced by Algorithm 3.2 provide an orthonormal basis for the wavelet space $W$, if $p_{\mu v}(\mu \in \mathscr{E} \backslash\{0\}, v \in \mathscr{E})$ are chosen as follows:

$$
p_{\mu \nu}=\left\{\begin{array}{lll}
-\bar{p}_{\mu} \sigma\left(p_{0}\right), & \text { for } & v=0 \\
\delta_{\mu v}-\bar{p}_{\mu} p_{v} /\left(1+\left|p_{0}\right|\right), & \text { for } & \nu \in \mathscr{E} \backslash\{0\}
\end{array}\right.
$$

While Theorem 3.5. gives a very general result, it has a serious shortcoming. The decay rates of the wavelets $\psi_{\mu}(\mu \in \mathscr{E} \backslash\{0\})$ are beyond our control, even when the scaling function $\phi$ has a certain decay rate. Let us take a closer look into this problem. If we want the sequences $b_{\mu \nu}$ in Algorithm 3.2 to be in $l_{1}\left(\mathbb{Z}^{d}\right)$, then the functions $p_{\mu \nu}$ must be continuous. But the discontinuous function $\sigma$ was used in the above construction. It will be demonstrated in Section 4 that the use of discontinuous functions is unavoidable if the information about the intrinsic properties of the scaling function $\phi$ is not available. Nevertheless, if some intrinsic properties of the scaling function $\phi$ are known, then it is possible to construct orthogonal wavelets having the same decay rates as $\phi$ does. The following example illustrates this point.

Example 3.6. Let $\phi$ be a function satisfying (2.6) and (3.2). Suppose in addition that $\hat{\phi}$ is nonnegative almost everywhere. Then there exists a null set $E \subset \mathbb{R}^{d}$ such that $[\hat{\phi}, \hat{\phi}](\xi)>0$ and $\hat{\phi}(\xi) \geqq 0$ for all $\xi \in \mathbb{R}^{d} \backslash E$. It follows from (3.4) that

$$
\hat{\phi}(\xi)=\hat{\phi}(\xi / 2) p\left(e^{-i \xi / 2}\right)
$$

where

$$
p\left(e^{-i \xi / 2}\right)=2^{-d / 2} \sum_{v \in \delta} e^{-i v \cdot \xi / 2} p_{v}\left(e^{-i \xi}\right)
$$

Let $F:=(E+2 \pi \mathbb{Z}) \cup 2(E+2 \pi \mathbb{Z})$. Then $F$ is also a null set. If $\xi \in \mathbb{R}^{d} \backslash F$, we have $\hat{\phi}(\xi / 2+2 \pi \alpha) \geqq 0$ for all $\alpha \in \mathbb{Z}^{d}$ and $[\hat{\phi}, \hat{\phi}](\xi / 2)>0$; hence $\hat{\phi}(\xi / 2+2 \pi \beta)>0$ for some $\beta \in \mathbb{Z}^{d}$. With this $\beta$ we deduce from (3.13) that

$$
\hat{\phi}(\xi+4 \pi \beta)=\hat{\phi}(\xi / 2+2 \pi \beta) p\left(e^{-i \xi / 2}\right) .
$$

Since $\xi \notin(E+2 \pi \mathbb{Z})$, we also have $\hat{\phi}(\xi+4 \pi \beta) \geqq 0$. This shows that $p\left(e^{-i \xi / 2}\right) \geqq 0$ for almost every $\xi \in \mathbb{R}^{d}$. Now (3.14) tells us that for almost every $\xi \in \mathbb{R}^{d}$,

$$
p_{0}\left(e^{-i \xi / 2}\right)=2^{-d / 2} \sum_{\mu \in \zeta} \sum_{\nu \in \zeta} e^{-i v(\xi / 2+\pi \mu)} p_{v}\left(e^{-i \xi}\right)=\sum_{\mu \in \zeta} p\left(e^{-i(\xi+2 \pi \mu) / 2}\right) \geqq 0 .
$$

Consequently, $\sigma\left(p_{0}\right)=1$ and $\left|p_{0}\right|=p_{0}$. Therefore $p_{\mu \nu}$ are continuous functions as long as $p_{v}$ are. Furthermore, if $\phi$ has orthonormal shifts and decays exponentially fast, then the wavelets $\psi_{\mu}(\mu \in \mathscr{E} \backslash\{0\})$ provided by Algorithm 3.2 also decay exponentially fast.

In the rest of this section we extend the preceding discussion to wavelet decompo- 
sitions generated by a finite number of functions. This problem was studied by Goodman, Lee, and Tang [18] for the univariate situation, and by Micchelli [35] for the multivatiate situation. Let $S=\mathscr{S}(\Phi)$ be an FSI subspace of $L_{2}\left(\mathbb{R}^{d}\right)$. We assume that the Gram matrix $G_{\Phi}:=([\hat{\phi}, \hat{\chi}])_{\phi . x \in \Phi}$ is nonsingular almost everywhere. This assumption covers all the cases considered in [18] and [35]. From the general theory of shift invariant-spaces developed in [5], we see that there exists a finite subset $\Phi_{*}$ of $L_{2}\left(\mathbb{R}^{d}\right)$ such that $\mathscr{S}\left(\Phi_{*}\right)=\mathscr{S}(\Phi)$ and the shifts of the functions in $\Phi_{*}$ are orthonormal. Thus, we may assume from the beginning that $\Phi$ has this property.

Theorem 3.7. Let $\Phi$ be a subset of $L_{2}\left(\mathbb{B}^{d}\right)$ containing $n$ elements such that the shifts of the functions in $\Phi$ are orthonormal. If, in addition, $\mathscr{S}(\Phi)$ is refinable, then there exists a set $\Psi$ containing $\left(2^{d}-1\right) n$ elements such that $\Psi$ provides an orthonormal basis for the wavelet space $W$.

Sketch of proof. As was done before, this problem can be reduced to a problem of matrix extension. Let $\Gamma:=\Phi \times \mathscr{E}$. For $\gamma=(\phi, \mu) \in \Phi \times \mathscr{E}=\Gamma$, let

$$
\rho_{y}:=2^{d / 2} \phi(2 \cdot-\mu) \text {. }
$$

Then $\left\{\rho_{y}: \gamma \in \Gamma\right\}$ provides an orthonormal basis for $S_{1}$, the dyadic dilate of $\mathscr{S}(\Phi)$. Each $f \in S_{1}$ can be represented as

$$
f=\sum_{\gamma \in \Gamma} \rho_{\gamma^{* \prime}} b_{f, \gamma}
$$

where $b_{f, \gamma}$ are sequences in $l_{2}\left(\mathbb{Z}^{d}\right)$. Let $p_{f, \gamma}$ be the symbol of the sequence $b_{f, \gamma}$ for each pair $(f, \gamma)$. We associate to each $f \in S_{1}$ a vector-valued function $p_{f}:=\left(p_{f, \gamma}\right)_{\gamma \in \Gamma}$ defined on $\mathbb{J}^{d}$. In particular, each $\phi \in \Phi$ is associated with $p_{\phi}: \mathbb{T}^{d} \rightarrow \mathbb{C}^{\Gamma}$. Since the shifts of the functions in $\Phi$ are orthonormal, $\left\{p_{\phi}(z): \phi \in \Phi\right\}$ is an orthonormal set in $\mathbb{C}^{r}$ for almost every $z \in \mathbb{T}^{d}$. A subset $\Psi$ of $S_{1}$ provides an orthonormal basis for the wavelet space $W$ if and only if

$$
\left\{p_{\phi}(z): \phi \in \Phi\right\} \cup\left\{p_{\psi}(z): \psi \in \Psi\right\}
$$

forms an orthonormal basis for $\mathbb{C}^{\Gamma}$ for almost every $z \in \mathbb{T}^{d}$. Thus, in order to establish the theorem, it suffices to prove the following lemma.

Lemma 3.8. Let $v_{1}, \ldots, v_{m}(m<n)$ be vector-valued measurable functions, i.e., $v_{j}=$ $\left(v_{j 1}, \ldots, v_{j n}\right)^{T}$ with $v_{j k}(k=1, \ldots, n)$ being complex-valued measurable functions on $a$ measurable space $(X, \mathscr{M})$. Suppose that $\left\{v_{1}(x), \ldots, v_{m}(x)\right\}$ is an orthonormal set in $\mathbb{C}^{n}$ for almost every $x \in X$. Then one can find vector-valued functions $v_{m+1}, \ldots, v_{n}$ such that for almost every $x \in X$, the vectors $v_{1}(x), \ldots, v_{n}(x)$ from an orthonormal basis for $\mathbb{C}^{n}$.

This lemma can be proved as follows. Choose a measurable vector-valued function $v_{m+1}=\left(v_{m+1,1}, \ldots, v_{m+1, n}\right)^{T}$ from $(X, \mathscr{M})$ to $\mathbb{C}^{n}$ such that for all $x \in X$, 


$$
\sum_{k=1}^{n}\left|v_{m+1, k}(x)\right|^{2}=1 \quad \text { and } \quad v_{j}^{*}(x) v_{m+1}(x)=0, \quad j=1, \ldots, m
$$

The existence of $v_{m+1}$ is guaranteed by Lemma 2.4. Continuing in this way, one can construct $v_{m+1}, \ldots, v_{n}$ so that they satisfy the requirements of the lemma.

\section{Extensibility}

Having reduced the problem of wavelet decomposition to that of matrix extension, we shall devote this section to a study of the extensibility in an abstract setting. It turns out that this problem is closely related to the theory of vector fields on spheres.

Given a commutative ring $R$ with identity, let $R^{n}$ be the free $R$-module of rank $n \geqq 2$. We say that an element $\left(p_{1}, \ldots, p_{n}\right) \in R^{n}$ is extensible over $R$, if $\left(p_{1}, \ldots, p_{n}\right)$ is the first row of some $n \times n$ invertible matrix over $R$. We are particularly interested in the rings of real-valued or complex-valued continuous functions on unit spheres.

For an integer $n \geqq 2$, let $S^{n-1}$ be the $(n-1)$-dimensional sphere

$$
\left\{\left(x_{0}, \ldots, x_{n-1}\right) \in \mathbb{R}^{n}: \sum_{j=0}^{n-1} x_{j}^{2}=1\right\}
$$

Let $R$ be the ring of all real-valued continuous functions on $S^{n-1}$. Consider the functions $p_{k} \in R$ given by

$$
p_{k}(x)=x_{k}, \quad k=0, \ldots, n-1
$$

The row vector $\left(p_{0}, \ldots, p_{n-1}\right)$ is extensible over $R$ only if $n=2,4$, or 8 . This conclusion comes from Adams' theorem (see [1]). Let $\rho(n)-1$ be the maximal number of linearly independent tangent vector fields on $S^{n-1}$. Write $n$ as $n=a(n) 2^{c(n)} 16^{d(n)}$, where $a(n), c(n)$, and $d(n)$ are nonnegative integers, $a(n)$ is odd, and $0 \leqq c(n) \leqq 3$. Adams' theorem says that

$$
\rho(n)=2^{c(n)}+8 d(n)
$$

Since $\left(p_{0}, \ldots, p_{n-1}\right)$ is extensible over $R$ if and only if $\rho(n)=n$, Adams' theorem tells us that this happens if and only if $n \in\{2,4,8\}$. For $n \in\{2,4,8\}$, it is not difficult to complete $\left(p_{0}, \ldots, p_{n-1}\right)$ to an orthogonal matrix. For example, one can find such a construction from [28, p. 31].

We remark that there is an oversight in $[33$, p. 92] (see also [16, p. 319]) concerning vector fields on spheres. It was stated there that there exists no nowhere-vanishing continuous vector fields tangent to the unit sphere $S^{n-1}$ except in real dimension 2,4 , or 8. In fact, the formula (4.1) tells us that for any even number $n$, there exists at least one continuous nowhere-vanishing vector field tangent to the unit sphere $S^{n-1}$. 
Next, let us consider the ring of the complex-valued continuous functions on the sphere

$$
S_{\mathrm{C}}^{n-1}:=\left\{\left(z_{0}, \ldots, z_{n-1}\right) \in \mathbb{C}^{n}: \sum_{j=0}^{n-1}\left|z_{j}\right|^{2}=1\right\}
$$

Let $f_{0 k}(k=0, \ldots, n-1)$ be the function defined by the rule

$$
f_{0 k}\left(z_{0}, \ldots, z_{n-1}\right)=z_{k}, \quad\left(z_{0}, \ldots, z_{n-1}\right) \in S_{\mathrm{C}}^{n-1} .
$$

If $n=2$, the $2 \times 2$ matrix

$$
\left(\begin{array}{cc}
z_{1} & z_{2} \\
-\bar{z}_{2} & \bar{z}_{1}
\end{array}\right)
$$

is unitary for every $\left(z_{1}, z_{2}\right) \in \mathbb{C}^{2}$ with $\left|z_{1}\right|^{2}+\left|z_{2}\right|^{2}=1$. The following theorem deals with the case $n>2$.

Theorem 4.1. If $n>2$, then there do not exist continuous functions $f_{j k}(j=1, \ldots, n-1$, $k=0, \ldots, n-1)$ on $S_{\mathrm{C}}^{n-1}$ such that the matrix $F(z):=\left(f_{j k}(z)\right)_{0 \leqq j, k \leqq n-1}$ is invertible for every $z \in S_{\mathrm{C}}^{n-1}$.

Proof. This theorem can be derived from Corollary (3.7) on page 24 of [21]. Here we give a proof based on Adam's theorem which works for all cases but $n=4$.

Suppose that there exist continuous functions $f_{j k}$ on $S_{\mathrm{C}}^{n-1}(j=1, \ldots, n-1$; $k=0, \ldots, n-1)$ such that the matrix $F(z)=\left(f_{j k}(z)\right)_{0 \leqq j, k \leqq n-1}$ is invertible for every $z \in S_{\mathrm{C}}^{n-1}$. Write $z_{j}=x_{j}+i y_{j}$ for $x_{j}, y_{j} \in \mathbb{R}$. Then $z=\left(z_{0}, \ldots, z_{n-1}\right) \in S_{\mathrm{C}}^{n-1}$ if and only if $\left(x_{0}, y_{0}, \ldots, x_{n-1}, y_{n-1}\right) \in S^{2 n-1}$. Let

$$
u:=\left(w_{0}, \ldots, w_{2 n-1}\right):=\left(x_{0}, y_{0}, \ldots, x_{n-1}, y_{n-1}\right)
$$

Thus, $f_{j k}(j, k=0, \ldots, n-1)$ may be viewed as functions of $w$ on $S^{2 n-1}$. Write $f_{j k}=g_{j k}+i h_{j k}$, where $g_{j k}$ and $h_{j k}$ are the real and imaginary parts of $f_{j k}$, respectively. In particular, $g_{0 k}=x_{k}, h_{0 k}=y_{k}(k=0, \ldots, n-1)$. Consider the matrix

$$
G:=\left(G_{j k}\right)_{0 \leqq j, k \leqq n-1},
$$

where each $G_{j k}$ is a $2 \times 2$ block:

$$
G_{j k}:=\left(\begin{array}{cc}
g_{j k} & h_{j k} \\
-h_{j k} & g_{j k}
\end{array}\right)
$$

We claim that 


$$
\operatorname{det} G(w)=0 \Rightarrow \operatorname{det} F(z)=0 .
$$

In order to verify this claim, we fix $z \in S_{\mathbf{c}}^{n-1}$ for the time being. Clearly, $\operatorname{det} G(w)=0$ implies that there exist $\xi_{j}, \eta_{j} \in \mathbb{R}(j=0, \ldots, n-1)$, not all zero, such that

$$
\sum_{j=0}^{n-1}\left(\xi_{j}, \eta_{j}\right) G_{j k}(w)=0 \text { for all } k=0, \ldots, n-1 .
$$

It follows that

$$
\sum_{j=0}^{n-1}\left(\xi_{j} g_{j k}(w)-\eta_{j} h_{j k}(w)\right)=0 \text { and } \sum_{j=0}^{n-1}\left(\xi_{j} h_{j k}(w)+\eta_{j} g_{j k}(w)\right)=0 .
$$

Let $\zeta_{j}:=\xi_{j}+i \eta_{j}$. Then $\left(\zeta_{0}, \ldots, \zeta_{n-1}\right) \in \mathbb{C}^{n} \backslash\{0\}$. Moreover,

$$
\sum_{j=0}^{n-1} \zeta_{j} f_{j k}(z)=0 \text { for all } k=0, \ldots, n-1 .
$$

Hence $\operatorname{det} F(z)=0$. This verifies our claim. Thus, if $\operatorname{det} F(z) \neq 0$ for every $z \in S_{\mathrm{C}}^{n-1}$, then $\operatorname{det} G(w) \neq 0$ for every $w \in S^{2 n-1}$. It follows that $\left(w_{0}, \ldots, w_{2 n-1}\right)$ is extensible over the ring of real-valued continuous functions on $S^{2 n-1}$. By Adams' theorem, this happens only if $2 n \in\{4,8\}$, i.e., $n \in\{2,4\}$.

In the case $n=4$ or 8 , however, if a vector-valued function $\left(f_{00}, \ldots, f_{0, n-1}\right)$ on $S_{\mathrm{c}}^{n-1}$ enjoys a certain symmetry, then it is possible to complete such a vector to a nonsingular matrix. Here we describe without proof a construction essentially given by Riemenschneider and Shen (see [37] and [38]). A mapping $\eta$ from $\mathscr{E}_{d}$ to itself is called admissible if $\eta$ satisfies the following two conditions:

$$
\eta(0)=0
$$

and

$$
(\eta(\mu)+\eta(v)) \cdot(\mu+v) \text { is odd for } \mu \neq v, \mu, v \in \mathscr{E}_{d} .
$$

Admissible mappings on $\mathscr{E}_{d}$ were constructed in [37] for $d \leqq 3$. When $d>3$, there do not exist admissible mappings on $\mathscr{E}_{\mathrm{d}}$. Suppose $\zeta_{v}\left(v \in \mathscr{E}_{\mathrm{d}}\right)$ are $2^{d}$ complex numbers. For any $\alpha \in \mathbb{Z}^{d}$, there exists a unique $\mu \in \mathscr{E}_{d}$ such that $\alpha-\mu \in 2 \mathbb{Z}^{d}$, we define $\zeta_{a}:=\zeta_{\mu}$.

Theorem 4.2. Let $c \in \mathbb{Z}^{d}$ be given, and let $\eta$ be an admissible mapping on $\mathscr{E}=\mathscr{E}_{d}$. Suppose $\zeta_{v}(v \in \mathscr{E})$ are $2^{d}$ complex numbers such that

$$
\sum_{v \in \zeta}\left|\zeta_{v}\right|^{2}=1
$$


and

$$
\bar{\zeta}_{v}=\zeta_{c-v} \text { for all } v \in \mathscr{E}
$$

Let

$$
w_{\mu v}:=\left\{\begin{array}{lll}
(-1)^{\eta(\mu) \cdot v \zeta_{v-\mu},} & \text { if } \eta(\mu) \cdot c \text { is even } \\
(-1)^{\eta(\mu) \cdot v \zeta_{v-\mu},} & \text { if } & \eta(\mu) \cdot c \text { is odd }
\end{array}\right.
$$

Then $\left(w_{\mu v}\right)_{\mu, v \in \delta}$ is a unitary matrix with $\left(\zeta_{v}\right)_{v \in \delta}$ as its first row.

\section{Construction of wavelets and prewavelets}

The results in the previous section tell us that, in general, the intrinsic properties of the scaling function must be employed in order to construct wavelets with certain decay rates. In this section we are particularly interested in symmetric scaling functions. The property of a scaling function being symmetric has some nice consequences and enables us to give a simple and explicit construction of wavelets and prewavelets. We also discuss the construction of box spline wavelets in arbitrary dimension.

By virtue of Algorithm 3.2 we may concentrate on the problem of completing a given vector to a nonsingular matrix. Let $p_{1}, \ldots, p_{n}$ be complex-valued continuous functions on $\mathbb{T}^{d}$ such that

$$
\sum_{j=1}^{n}\left|p_{j}(z)\right|^{2}=1 \quad \text { for all } z \in \mathbb{T}^{d}
$$

Consider the mapping

$$
P: z \mapsto\left(p_{1}(z), \ldots, p_{n}(z)\right), \quad z \in \mathbb{T}^{d} .
$$

Then $P$ maps $\mathbb{J}^{d}$ to $S_{\mathrm{C}}^{n-1}$. If the functions $p_{1}, \ldots, p_{n}$ are Hölder continuous on $\mathbb{T}^{d}$, then $P$ is a Hölder continuous mapping on $\mathbb{T}^{d}$; that is, there are some $\kappa \in(0,1]$ and a constant $C>0$ such that

$$
|P(z)-P(w)| \leqq C|z-w|^{\kappa} \quad \text { for all } z, w \in \mathbb{T}^{d}
$$

The Hausdorff dimension of $\mathbb{T}^{d}$ and $S_{\mathrm{c}}^{n-1}$ is $d$ and $2 n-1$, respectively. Thus, by a version of the Sard theorem (see e.g., [26]), the image of the mapping $P$ is a proper subset of $S_{\mathrm{C}}^{n-1}$ provided $d<2 n-1$. In other words, if $d<2 n-1$, then there exists a point in $S_{\mathrm{C}}^{n-1}$ outside the range of $P$. After an appropriate rotation if necessary, we may assume that this point is $-e_{1}$, where $e_{1}$ denotes the $n$-vector $(1,0, \ldots, 0)$.

Now we are in a position to describe the results of Gröchenig [19] and Meyer [33, p. 92] in a slightly different way (also see [39]). Let $p_{1}, \ldots, p_{n}$ be complex-valued 
continuous functions on $T^{d}$ satisfying the condition (5.1). If $\left(p_{1}(z), \ldots, p_{n}(z)\right) \neq-e_{1}$ for any $z \in \mathbb{T}^{d}$, then for sufficiently small $\varepsilon>0$,

$$
\operatorname{det}\left[\begin{array}{cccc}
p_{1}(z) & p_{2}(z) & \ldots & p_{n}(z) \\
-\bar{p}_{2}(z) & \varepsilon & \cdots & 0 \\
\vdots & \vdots & \ddots & \vdots \\
-\bar{p}_{n}(z) & 0 & \cdots & \varepsilon
\end{array}\right] \neq 0 \quad \text { for any } z \in \mathbb{T}^{d}
$$

Then the Gram-Schmidt orthogonalization procedure is performed to obtain a unitary matrix with $\left(p_{1}(z), \ldots, p_{n}(z)\right)$ as its first row.

A different construction was given in [26]. The following theorem is a slight modification of [26, Prop. 2.1].

Theorem 5.1. Let $I$ be the $n \times n$ identity matrix, e the $n \times 1$ vector $(1,0, \ldots, 0)^{T}$, and $t a$ complex number with $|t|=1$. For $z=\left(z_{1}, \ldots, z_{n}\right)^{T} \in S_{\mathrm{C}}^{n-1} \backslash\{$ te $\}$, let

$$
Q(z):=t I-(\bar{t} e-\bar{z})(t e-z)^{T} /\left(\bar{t}-\bar{z}_{1}\right) .
$$

Then $Q(z)$ is a unitary matrix with $\left(z_{1}, \ldots, z_{n}\right)$ as its first row.

Proof. First we compute $(t e-z)^{T}(\bar{t} e-\bar{z})$ as follows:

$$
(t e-z)^{T}(t e-\bar{z})=1-\bar{t} z_{1}-t \bar{z}_{1}+1=2-t z_{1}-t \bar{z}_{1} .
$$

It follows that

$$
Q(z)(Q(z))^{*}=I+(\overline{t e}-\bar{z})(t e-z)^{T}\left(\frac{2-\bar{t} z_{1}-t \bar{z}_{1}}{\left|t-z_{1}\right|^{2}}-\frac{t}{t-\bar{z}_{1}}-\frac{t}{t-z_{1}}\right)=I
$$

Hence $Q(z)$ is a unitary matrix. Moreover, the $(1,1)$-entry of $Q(z)$ is

$$
t-\left(\bar{t}-\bar{z}_{1}\right)\left(t-z_{1}\right) /\left(\bar{t}-\bar{z}_{1}\right)=z_{1},
$$

and the $(1, k)$-entry $(k>1)$ is $\left(t-\bar{z}_{1}\right) z_{k} /\left(t-\bar{z}_{1}\right)=z_{k}$.

We contend that the construction given in Theorem 5.1 has two advantages over those given by Gröchenig and Meyer. First, the matrix given in Theorem 5.1 is already, unitary, so no further orthogonalization procedure is needed. Second, the matrix in (5.2) involves $\varepsilon$, which depends on the magnitude of the functions $p_{j}(j=1, \ldots, n)$. In contrast to this inconvenience, the construction given in (5.3) is universal, as long as $z \in S_{\mathrm{C}}^{n-1} \backslash$ $\{a e\}$.

Theorem 5.1 can be applied to symmetric scaling functions. Given a function $\phi \in L_{2}\left(\mathbb{R}^{d}\right)$, we say that $\phi$ is skew-symmetric about some point $c_{\phi} \in \mathbb{R}^{d}$ if 


$$
\phi\left(c_{\phi}+\cdot\right)=\bar{\phi}\left(c_{\phi}-\cdot\right) \text {. }
$$

If, in addition, $\phi$ is refinable and $\hat{\phi} \neq 0$ a.e., then $c_{\phi}$ must lie in $\mathbb{Z}^{d} / 2$ (see [15], [8], and [38]). Without loss of generality, we may assume that $c_{\phi} \in \mathscr{E}_{d} / 2$. Let $c:=2 c_{\phi}$. Then $c \in \mathscr{E}_{d}$ and $\phi=\phi(c-\cdot)$. Suppose that $\phi$ has stable shifts and satisfies the refinement equation (3.2). Then we have

$$
\begin{aligned}
\phi & =\phi(c-\cdot)=\sum_{\alpha \in \mathbf{Z}^{d}} b(\alpha) \phi(2 c-2 \cdot-\alpha) \\
& =\sum_{\alpha \in \mathbf{Z}^{d}} b(\alpha) \phi(2 \cdot+\alpha-c)=\sum_{\alpha \in \mathbf{Z}^{d}} b(c-\alpha) \phi(2 \cdot-\alpha) .
\end{aligned}
$$

Comparing (5.4) with (3.2), we find

$$
b(\alpha)=b(c-\alpha) \quad \text { for all } \alpha \in \mathbb{Z}^{d} .
$$

For $\mu \in \mathbb{Z}^{d}$, let

$$
p_{\mu}(z)=\sum_{\beta \in \mathbb{Z}^{d}} 2^{-d / 2} b(\mu+2 \beta) z^{\beta}, \quad z \in \mathbb{T}^{d} .
$$

Then (5.5) implies that for all $\mu \in \mathbb{Z}^{d}$,

$$
\begin{aligned}
p_{\mu}(z) & =\sum_{\beta \in Z^{d}} 2^{-d / 2} b(\mu+2 \beta) z^{\beta}=\sum_{\beta \in Z^{d}} 2^{-d / 2} \bar{b}(c-\mu-2 \beta) z^{\beta} \\
& =\sum_{\beta \in Z^{d}} 2^{-d / 2} \overline{b(c-\mu-2 \beta) z^{-\beta}}=\overline{p_{c-\mu}(z)}, \quad z \in \mathbb{U}^{d} .
\end{aligned}
$$

When $d \leqq 3$, Riemenschneider and Shen ([37] and [38]) gave an explicit construction of orthogonal wavelet and prewavelet sets for the skew-symmetric scaling function $\phi$. In this section we consider the problem of constructing wavelets and prewavelets for the skew-symmetric scaling function $\phi$ in arbitrary dimension. Let $P$ be the mapping from $\mathbb{J}^{d}$ to $\mathbb{C}^{\delta}$ given by $z \mapsto\left(p_{\mu}(z)\right)_{\mu \in \varepsilon}$. Since $\phi$ has orthonormal shifts, (3.6) holds. In other words, the range of $P$ is contained in the sphere.

$$
\left\{\left(w_{\mu}\right)_{\mu \in \varepsilon} \in \mathbb{C}^{\delta}: \sum_{\mu \in \delta}\left|w_{\mu}\right|^{2}=1\right\}
$$

Let $e$ be the vector $\left(\delta_{0 v}\right)_{v \in \mathscr{E}}$. It follows from (5.7) that $p_{0}=\overline{p_{c}}$. Thus, if $c \in \mathscr{E} \backslash\{0\}$, then $-e \notin P\left(\mathbb{T}^{d}\right)$; if $c=0$, then $p_{0}$ is real, hence ie $\notin P\left(\mathbb{T}^{d}\right)$. Applying Theorem 5.1 to the current situation, we obtain the following results.

Theorem 5.2. Let $\phi \in L_{2}\left(\mathbb{R}^{d}\right)$ be a refinable function having orthonormal shifts. Let $p_{\mu}$ 
$(\mu \in \mathscr{E})$ be given as in (5.6). If $\phi$ is skew-symmetric about some point $c_{\phi} \in \mathscr{E}_{d} / 2 \backslash\{0\}$, then choosing

$$
p_{\mu v}= \begin{cases}\bar{p}_{\mu}\left(1+p_{0}\right) /\left(1+\bar{p}_{0}\right), & \text { for } \mu \in \mathscr{E} \backslash\{0\}, v=0 \\ -\delta_{\mu v}+\bar{p}_{\mu} p_{v} /\left(1+\bar{p}_{0}\right), & \text { for } \mu, v \in \mathscr{E} \backslash\{0\} .\end{cases}
$$

in Algorithm 3.2 produces an orthogonal wavelet set $\left\{\psi_{\mu}: \mu \in \mathscr{E} \backslash\{0\}\right\}$. If $\phi$ is skewsymmetric about the origin, then the choice

$$
p_{\mu \nu}= \begin{cases}-\bar{p}_{\mu}\left(i-p_{0}\right) /\left(i+\bar{p}_{0}\right), & \text { for } \mu \in \mathscr{E} \backslash\{0\}, v=0 \\ i \delta_{\mu \nu}+\bar{p}_{\mu} p_{v} /\left(i+\bar{p}_{0}\right), & \text { for } \mu, v \in \mathscr{E} \backslash\{0\}\end{cases}
$$

gives rise to an orthogonal wavelet set $\left\{\psi_{\mu}: \mu \in \mathscr{E} \backslash\{0\}\right\}$.

We note that the wavelets constructed here decay exponentially fast, if the scaling function $\phi$ does so.

If $\phi$ is a real-valued refinable function, then the refinement mask $b$ is real-valued. In Theorem 5.2, if $\phi$ is symmetric about $c_{\phi} \in \mathscr{E} / 2 \backslash\{0\}$, then the coefficient sequences $b_{\mu \nu}$ in the Laurent series of $p_{\mu \nu}(\mu, v \in \mathscr{E})$ are real, because $\overline{p_{\mu}}=p_{c-\mu}$. Hence the wavelets $\psi_{\mu}$ $(\mu \in \mathscr{E} \backslash\{0\})$ are real-valued. However, if $\phi$ is symmetric about the origin, the wavelets $\psi_{\mu}$ $(\mu \in \mathscr{E} \backslash\{0\})$ constructed in Theorem 5.2 are complex-valued in general. When $\phi$ is a box spline, the construction given in (5.8) is also valid for the latter case. Let us discuss this problem briefly.

Unconditional spline bases for function spaces were constructed by Ciesielski [11] and Strömberg [40] in the early 1980's. More recently, cardinal spline wavelets and prewavelets were studied in $[2,29,7,8$, and 34]. Box spline wavelets and prewavelets were investigated in $[37,38,25,9,30$, and 39]. Let us recall the definition of box splines (see [3]). Given a $d \times n$ integer matrix $X$ of rank $d \leqq n$, the box spline $B(\cdot \mid X)$ is defined by the equation

$$
\int_{\mathbb{R}^{d}} f(x) B(x \mid X) d x=\int_{[0,1]^{n}} f(X t) d t \text { for all } f \in C\left(\mathbb{R}^{d}\right) .
$$

Any box spline is refinable. Let $x_{1}, \ldots, x_{n}$ denote the columns of $X$. Then

$$
B(\cdot \mid X)=\sum_{\alpha \in Z^{d}} b(\alpha) B(2 \cdot-\alpha \mid X)
$$

with

$$
\tilde{b}(z):=\sum_{\alpha \in \mathbf{Z}^{d}} b(\alpha) z^{\alpha}=2^{-n+d} \prod_{j=1}^{n}\left(1+z^{x_{j}}\right) .
$$

It is well-known that the box spline $B(\cdot \mid X)$ has stable shifts if and only if $X$ is unimodular, i.e., any $d \times d$ submatrix of $X$ has determinant -1 , 0 , or 1 (see [14], [22], and [24]). Let $\therefore$

$$
c_{X}:=\sum_{j=1}^{n} x_{j} / 2
$$


be the centre of the box spline $B(\cdot \mid X)$. There is a unique vector $v_{X} \in \mathbb{Z}^{d}$ such that $c_{X}-v_{X} \in \mathscr{E}_{d} / 2$. Consider $\rho:=B\left(\cdot+v_{X} \mid X\right)$. Then $\rho$ is symmetric about $c_{X}-v_{X} \in \mathscr{E}_{d} / 2$. Suppose that the matrix is unimodular. Then $B(\cdot \mid X)$ has stable shifts, and $\mid \hat{\rho}, \hat{\rho}]>0$ everywhere on $\mathbb{R}^{d}$. Let $\phi$ be the function given by

$$
\hat{\phi}=\hat{\rho} /[\hat{\rho}, \hat{\rho}]^{1 / 2}
$$

Then $\phi$ has orthonormal shifts and $\mathscr{S}(\phi)=\mathscr{P}(\rho)$. Moreover, $\phi$ decays exponentially fast at infinity. The function $\phi$ is also refinable:

$$
\phi=\sum_{\alpha \in \mathbf{Z}^{d}} a(\alpha) \phi(2 \cdot-\alpha)
$$

where the mask $a$ is given by

$$
\tilde{a}\left(e^{-i \theta}\right)=\tilde{b}\left(e^{-i \theta}\right) e^{i v X \cdot \theta} \sqrt{[\hat{\rho}, \hat{\rho}](\theta) /[\hat{\rho}, \hat{\rho}](2 \theta)}, \quad \theta \in \mathbb{R}^{d},
$$

so it also decays exponentially fast (see [25]). For $\mu \in \mathbb{Z}^{d}$, let $a_{\mu}(\beta):=a(\mu+2 \beta)$ and

$$
p_{u}(z):=2^{-d / 2} \sum_{\alpha \in Z^{d}} a_{\mu}(\beta) z^{\beta}, \quad z \in \mathbb{T}^{d}
$$

Then $p_{\mu}(\mu \in \mathscr{E})$ satisfy (3.6). We claim that there is no $z \in \mathbb{J}^{d}$ such that $p_{\mu}(z)=-\delta_{0 \mu}$ for all $\mu \in \mathscr{E}$. If $c_{X} \notin \mathbb{Z}^{d}$, then $c_{X}-v_{X} \in \mathscr{E}_{d} / 2 \backslash\{0\}$, so our claim is justified because (5.7) is valid with $c:=2\left(c_{X}-v_{X}\right)$. If $c_{X} \in \mathbb{Z}^{d}$, then $v_{X}=c_{X}$. In this case, $\tilde{b}\left(e^{-i \theta}\right) e^{i c X \cdot \theta}$ are real numbers for $\theta \in \mathbb{R}^{d}$. Moreover, it was proved by Stöckler [39] that there is no $\theta \in \mathbb{R}^{d}$ such that

$$
\tilde{b}\left(e^{-i(\theta+v \pi)}\right) e^{i c x \cdot(\theta+v \pi)}<0 \text { for all } v \in \mathscr{E} .
$$

Hence there is no $\theta \in \mathbb{R}^{d}$ such that

$$
\tilde{a}\left(e^{-i(\theta+v \pi)}\right)<0 \quad \text { for all } v \in \mathscr{E} .
$$

If there were some $z=e^{-i \theta}\left(\theta \in \mathbb{R}^{d}\right)$ such that $p_{\mu}(z)=-\delta_{0 \mu}$, then we would have

$$
\tilde{a}(z)=2^{d / 2} \sum_{\mu \in \delta} p_{\mu}\left(z^{2}\right) z^{\mu}=2^{d / 2} p_{0}\left(z^{2}\right)
$$

and (5.11) would follow. This contradiction verifies our claim. Consequently, Theorem 5.1 is applicable and we have the following results.

Theorem 5.3. Let $B(\cdot \mid X)$ be the box spline defined by a unimodular integer $d \times n$ matrix $X$. Let $\phi$ be the function derived from $B(\cdot \mid X)$ as given in (5.9), and $\phi_{\mu}=\phi(2 \cdot-\mu)$ $(\mu \in \mathscr{E})$. For $\mu, v \in \mathscr{E}$, let $p_{\mu \nu}$ be given as in (5.8), where $p_{\mu}$ are given as in (5.10), and let $a_{\mu \nu}$ be the coefficient sequences in the Laurent expansions of $p_{\mu v}$. Then the functions 


$$
\psi_{\mu}:=\sum_{v \in \mathscr{C}} \phi_{v}^{*^{\prime}} a_{\mu v}, \quad \mu \in \mathscr{E} \backslash\{0\},
$$

constitute an orthogonal set of wavelets with exponential decay.

In the rest of this section we study the construction of prewavelet sets for symmetric scaling functions. Let us recall from [25] the general procedure for the construction of prewavelet sets.

Algorithm 5.4. Let $\phi$ be a completely supported function having stable shifts. Assume that $\phi$ satisfies the refinement equation (3.2) with a finitely supported sequence $b$ as the refinement mask.

Step 1. Find $p_{\mu}(\mu \in \mathscr{E})$ by the equation $p_{\mu}(z):=\sum_{\beta \in Z^{d}} b(2 \beta+\mu) z^{\beta}, z \in \mathbb{T}^{d}$.

Step 2. Find Laurent polynomials $p_{\mu v}(\mu, \nu \in \mathscr{E})$ such that $p_{0 v}=p_{v}$ for all $v \in \mathscr{E}$ and that the matrix $\left(p_{\mu v}(z)\right)_{\mu, v \in \varepsilon}$ is nonsingular for every $z \in \mathbb{T}^{d}$.

Step 3. Let $\rho_{\mu}:=\sum_{v \in 6} \phi_{v}{ }^{\prime} b_{\mu v}$, where $b_{\mu v}$ are the coefficient sequences in the Laurent polynomials $p_{\mu v}$, and $\phi_{v}=\phi(2 \cdot-v)$.

Step 4. Set $\widehat{\psi}_{\mu}:=[\hat{\phi}, \hat{\phi}] \hat{\rho}_{\mu}-\left[\hat{\rho}_{\mu}, \hat{\phi}\right] \hat{\phi}, \mu \in \mathscr{E} \backslash\{0\}$.

Then $\psi_{\mu}(\mu \in \mathscr{E} \backslash\{0\})$ are compactly supported and provide a stable basis for the wavelet space $W$.

In order to apply Algorithm 5.4 to symmetric scaling functions, we first establish some elementary results on determinants.

Lemma 5.5. Let $\left(a_{1}, \ldots, a_{n}\right) \in \mathbb{C}^{n} \backslash\{0\}$. If $a_{1}$ is a real number, then

$$
\left|\begin{array}{cccc}
a_{1} & a_{2} & \ldots & a_{n} \\
i \bar{a}_{2} & 1 & \ldots & 0 \\
\vdots & \vdots & \ddots & \vdots \\
i \bar{a}_{n} & 0 & \ldots & 1
\end{array}\right| \neq 0
$$

If $a_{1}=\overline{a_{2}}$, then

$$
\left|\begin{array}{ccccc}
a_{1} & a_{2} & a_{3} & \ldots & a_{n} \\
1-\left(a_{1}+a_{2}\right) / 2 & 1+\left(a_{1}+a_{2}\right) / 2 & 0 & \ldots & 0 \\
-\bar{a}_{3} & \bar{a}_{3} & 1 & \ldots & 0 \\
\vdots & \vdots & \vdots & \ddots & \vdots \\
-\bar{a}_{n} & \bar{a}_{n} & 0 & \ldots & 1
\end{array}\right| \neq 0 .
$$

Proof. The first determinant

$$
\Delta_{1}=a_{1}-i\left(\left|a_{2}\right|^{2}+\cdots+\left|a_{n}\right|^{2}\right)
$$


hence

$$
\left|\Delta_{1}\right|^{2}=\left|a_{1}\right|^{2}+\left(\left|a_{2}\right|^{2}+\cdots+\left|a_{n}\right|^{2}\right)^{2}>0
$$

Adding the first column to the second column in the second determinant $\Delta_{2}$, we obtain

$$
\Delta_{2}=a_{1}-a_{2}+\left(a_{1}+a_{2}\right)^{2} / 2+2 \sum_{j=3}^{n}\left|a_{j}\right|^{2}=2 i \operatorname{Im} a_{1}+2\left(\operatorname{Re} a_{1}\right)^{2}+2 \sum_{j=3}^{n}\left|a_{j}\right|^{2}
$$

It follows that $\Delta_{2} \neq 0$.

Lemma 5.5 yields the following construction of prewavelets for symmetric functions.

Theorem 5.6. Let $\phi$ be a function satisfying all the conditions of Algorithm 5.4 and, in addition, is skew-symmetric about some $c_{\phi} \in \mathscr{E}_{d} / 2$. Then Algorithm 5.4 produces a prewavelet set of compactly supported functions if the Laurent polynomial $p_{\mu \nu}(\mu, v \in \mathscr{E})$ are chosen according to the following rule. If $c_{\phi}=0$, choose

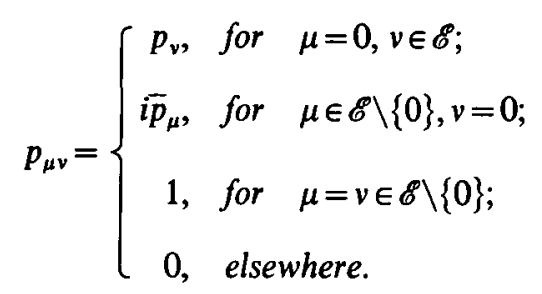

If $c_{\phi} \neq 0$, let $c=2 c_{\phi}$ and choose

$$
p_{\mu v}=\left\{\begin{array}{rll}
p_{v}, & \text { for } \mu=0, v \in \mathscr{E} ; \\
1-\left(p_{0}+p_{c}\right) / 2, & \text { for } \mu=c, v=0 \\
1+\left(p_{0}+p_{c}\right) / 2, & \text { for } \mu=c, v=c ; \\
-\bar{p}_{\mu}, & \text { for } \mu \in \mathscr{E} \backslash\{0, c\}, v=0 ; \\
\bar{p}_{\mu}, & \text { for } \mu \in \mathscr{E} \backslash\{0, c\}, v=c ; \\
1, & \text { for } \mu=v \in \mathscr{E} \backslash\{0, c\} \\
0, & \text { elsewhere. }
\end{array}\right.
$$

Acknowledgement. We are grateful to G. Peschke and the anonymous referee for pointing out the reference [21] to us.

\section{REFERENCES}

1. J. F. AdAms, Vector fields on spheres, Ann. of Math. 75 (1962), 603-632. 
2. G. Batre, A block spin construction of ondelettes. Part I: Lemaire Functions, Comm. Math. Phys. 110 (1987), 601-615.

3. C. DE Boor and K. HöllıG, B-splines from parallelepipeds, J. Analyse Math. 42 (1982/3), 99-115.

4. C. DE BOOR, R. DeVore and A. Ron, Approximation from shift-invariant subspaces of $L_{2}\left(\mathbb{R}^{d}\right)$, (CMS-TSR University of Wisconsin-Madison 92-2, 1991).

5. C. DE BOOR, R. DEVORE and A. RON, The structure of finitely generated shift-invariant spaces in $L_{2}\left(\mathbb{R}^{d}\right)$, (CMS-TSR University of Wisconsin-Masison 92-8, 1992).

6. C. DE BOoR, R. DEVORE and A. Ron, On the construction of multivariate (pre) wavelets, Constr. Approx. 9 (1993), 123-166.

7. C. K. Chul and J. Z. WANG, On compactly supported spline wavelets and a duality principle, Trans. Amer. Math. Soc. 330 (1992), 903-915.

8. C. K. Chui and J. Z. WANG, A general framework of compactly supported splines and wavelets, J. Approx. Theory 71 (1992), 263-304.

9. C. K. Chui, J. StOCKLER and J. D. WARD, Compactly supported box spline wavelets, Approx. Theory Appl. 8 (1992), 77-100.

10. P. G. Ciarlet, Introduction to Numerical Linear Algebra and Optimisation (Cambridge, University Press, 1988).

11. Z. Clesielski, Equivalence, unconditionality and convergence a.e. of the spline bases in $L_{p}$ spaces, in Approximation Theory (Banach Center Publications, 4) 55-68.

12. A. CoHEN, Ondelettes, analysis multiresolutions et traitement numerique du signal (Ph.D. Thesis, Université de Paris IX (Dauphine), France, 1990).

13. W. Dahmen and A. Kunoth, Multilevel preconditioning (Preprint No. A-91-31, Fachbereich Mathematik, Freie Universität Berlin, 1991).

14. W. Dahmen amd C. A. Micchelli, Translates of multivariate splines, Linear Algebra Appl. 52/53 (1983), 217-234.

15. I. Daubechies, Orthonormal bases of compactly supported wavelets, Comm. Pure Appl. Math. 41 (1988), 909-996.

16. I. Daubechies, Ten Lectures on Wavelets (CBMF Conferences Series in Applied Mathematics, 61, SIAM, Philadelphia, 1992).

17. R. DeVore and B. Lucier, Wavelets, Acta Numerica 1 (1991), 1-56.

18. T. N. T. Goodman, S. L. LeE and W. S. WANG, Wavelets in wandering subspaces, Trans. Amer. Math. Soc. 338 (1993), 639-654.

19. K. Gröhenıg, Analyse multi-échelles et bases d'ondelettes, C. R. Acad. Sci. Paris Sér. I Math. 305(1) (1987), 13-15.

20. H. Helson, Lectures on Invariant Subspaces (Academic Press, New York, 1964).

21. I. M. James, The Topology of Stiefel Manifolds (LMS Lecture Note Series 24, Cambridge University Press, Cambridge, 1976).

22. R. Q. JiA, On the linear independence of translates of a box spline, J. Approx. Theory 40 (1984), 158-160.

23. R. Q. JIA, A Bernstein type inequality associated with wavelet decomposition, Constr. Approx. 9 (1993), 299-318. 
24. R. Q. JIA and C. A. Micchelu, On linear independence of integer translates of a finite number of functions (Research Report CS-90-10, 1990, University of Waterloo). A revised version appeared in Proc. Edinburgh Math. Soc. 36 (1992), 69-85.

25. R. Q. JIA and C. A. MICChelLI, Using the refinement equation for the construction of pre-wavelets II: Powers of two, in Curves and Surfaces (P. J. Laurent, A. Le Méhauté and L. L. Schumaker, eds.) Academic Press, New York, 1991), 209-246.

26. R. Q. JiA and C. A. MiCCHELLI, Using the refinement equation for the construction of pre-wavelets V: extensibility of trigonometric polynomials, Computing 48 (1992), 61-72.

27. R. Q. JIA and J. Z. WANG, Stability and linear independence associated with wavelet decompositions, Proc. Amer. Math. Soc. 117 (1993), 1115-1124.

28. T. Y. LAM, Serre's Conjecture (Lecture Notes in Math. 635, Springer-Verlag, New York, 1978).

29. P. G. Lemarié, Ondelettes à localisation exponentielle, J. Math. Pures Appl. 67 (1988), 227-236.

30. R. A. LORENTZ and W. R. MADYCH, Wavelets and generalized box splines, preprint.

31. W. R. MADYCH, Some elementary properties of multiresolution analyses of $L_{2}\left(\mathbb{R}^{n}\right)$, in Wavelets - A Tutorial in Theory and Applications (Academic Press, New York, 1992), 259-294.

32. S. G. Mallat, Multiresolution approximations and wavelet orthonormal bases of $L_{2}\left(\mathbb{R}^{n}\right)$, Trans. Amer. Math. Soc. 315 (1989), 69-87.

33. Y. MeYer, Ondelettes et Opérateurs I: in Ondelettes (Herman Éditeurs, 1990).

34. C. A. Micchelli, Using the refinement equation for the construction of pre-wavelets, Numerical Algorithms 1 (1991), 75-116.

35. C. A. MicChelLI, Using the refinement equation for the construction of pre-wavelets VI: shift-invariant subspaces, to appear in the Proceedings of the NATO ASI held in Maratea, Italy, April 1991.

36. C. A. Micchell, A tutorial on multivariate wavelet decomposition, to appear in the Proceedings of the NATO ASI held in Maratea, Italy, April 1991.

37. S. D. Riemenschneider and Z. W. Shen, Box splines, cardinal series and wavelets, in Approximation Theory and Functional Analysis (C. K. Chui, ed., Academic Press, 1991), 133-149.

38. S. D. RiemensChNEIDER and Z. W. SHEN, Wavelets and prewavelets in low dimensions, $J$. Approx. Theory 71 (1992), 18-38.

39. J. STÖOCKLeR, Multivariate wavelets, in Wavelets-A Tutorial in Theory and Applications (Academic Press, New York, 1992), 325-355.

40. J.-O. Strómberg, A modified Franklin system and higher-order spline system on $\mathbb{R}^{n}$ as unconditional bases for Hardy spaces, in Conference on Harmonic Analysis in Honor of Antoni Zygmund, Vol. II, W. Beckner et al., eds., Wadsworth, Belmont, California, 1983), 457-493.

41. N. Wiener, The Fourier Integral and Certain of its Applications (Dover Publications, Inc., New York, 1958).

Department of Mathematics

University of Alberta

Edmonton, Canada T6G 2G1
Center for Mathematical Sciences

UNIVERSITY OF WISCONSIN

MADison, WI 53705, U.S.A. 